\title{
Roles of Waxy and Soluble Starch Synthase lia Alleles in Determining Different Type Resistant Starch Contents of Rice
}

Hui You

Southwest University of Science and Technology

Ouling Zhang

Southwest University of Science and Technology

Xu Liang

Southwest University of Science and Technology

Cheng Liang

Southwest University of Science and Technology

Yongjun Chen

Southwest University of Science and Technology

Xunchao Xiang ( $\nabla$ xiangxunchao@swust.edu.cn )

southwest unversity of science and technology https://orcid.org/0000-0002-5926-3011

Original article

Keywords: Physicochemical properties, Rice (Oryza sativa L.), Resistant Starch, RS2, SSIla, Wx

Posted Date: November 19th, 2020

DOI: https://doi.org/10.21203/rs.3.rs-107470/v1

License: (c) (i) This work is licensed under a Creative Commons Attribution 4.0 International License.

Read Full License 


\section{Abstract}

\section{Background}

Resistant Starch (RS) is a functional starch that has functions of regulating diabetes, hypertension and obesity. The effects of most starch synthesis-related genes (SSRGs) on RS content and their relationships are largely unknown.

\section{Results}

In current study, ninety-nine lines from a recombinant inbred line were selected to investigate the effects of SSRGs on the RS content in different process status. Results revealed that RS content decreased dramatically after cooking, but it did not increase significantly after cooling for 7 days. And RS was closely related to many indexes of physicochemical properties, but was not correlated with granule size. Waxy $(W x)$ played an important role in controlling RS content and $W x^{a}$ could elevate RS content in raw milled rice, cooked rice and retrograded rice. Soluble starch synthase Ila (SSIla) had an impact on RS2, and RS2 content of indica SSIla were significantly higher than that of japonica SSIIa (SSIlaj). Moreover, interaction of $W x$ and SSIla was responsible for variations of RS content in three sample types, RS2 and volume proportion of different size starch granules.

\section{Conclusions}

$W x$ and SSIla together significantly regulate different types content of RS in rice, but SSIla only affects RS2. $W x^{a}$-SSIlaj is favorable to forming large-diameter starch granules.

\section{Background}

Resistant starch (RS) is a sum of starch or starchy food products that cannot be digested in the small intestine of healthy individuals but fermented in the large intestine by microbial flora (Asp 1992). According to the botanical origin and nature, RS can be furtherly classified into five subtypes: RS1, RS2, RS3, RS4 and RS5 (Xia et al. 2018). RS2 and RS3 are the main types found in raw and cooked rice, respectively. RS2 is made up of native starch granules whose formation and structure protects it from digestion and is usually present in raw potato, green banana and uncooked starch (Hernández et al. 2008). The physiological functions of RS2 are few because of its rapid digestion after being thermally processed and cooked. Because of the higher starch gelatinization temperature and lower viscosity, RS2 is often used to improve the gluten structure of biscuits, bread and other pasta products, to increase the product moisture content, and has certain commercial value (Korus et al. 2009). RS3 consists of retrograded starch, mainly the recrystallized amylose, formed during cooling of gelatinized starch and cooked foods that are stored at room or low temperature (Sharma et al. 2008), which is widely used in food processing industry. 
With the development of living standards and changes of life style, the number of people with type-2 diabetes are increasing. Consumption of food with high RS can help to prevent from diabetes, and there is a decrease in postprandial glucose and insulin responses because of its slow digestion and absorption by the small intestine (Raigond et al. 2015). Meanwhile, RS can be decomposed into short chain fatty acids such as acetic acid, propionic acid and butyric acid in the large intestine, which can improve the health of large intestine and prevent the occurrence of intestinal inflammatory diseases and colon cancer (Robertson et al. 2000; Topping et al. 2008). Consumption of RS can increase satiety and reduce calorie intake, which helps weight management (Raigond et al. 2015). Therefore, it is important to elevate the content of RS in food.

Rice (Oryza sativa L.) feeds more than half of the world's population as staple food and is the main source of nutrition and carbohydrates for many people. RS content is generally under $3 \%$ in hot cooked rice cultivars, which is not enough to confer the associated health benefits (Frei et al. 2003; Hu et al. 2004). Therefore, many scholars have focused on the promotion of RS content in rice varieties. In the past decades, many mutants or varieties with elevated RS have been identified, such as RS111 (Yang et al. 2006), Gaomi 2 (Kim et al. 2006), Jiangtangdao 1 (Yang et al. 2012) and b10 (Zhou et al. 2016).

Starch biosynthesis is a sophisticated process involving multiple enzymes, including ADP-glucose pyrophosphorylase (AGPase), granule-bound starch synthase (GBSS), soluble starch synthase (SS), starch branching enzymes (SBEs), isoamylase (ISA) and pullulanase (PUL) (Tian et al. 2009). Among them, GBSSI is encoded by the Waxy $(W x)$, which plays an important role in determining the synthesis of amylose. SS, SBEs, ISA and PUL can control the synthesis of amylopectin. RS content can be affected by the ratio of amylose to amylopectin and the fine structure of starch (Eggum et al. 1993; Hu et al. 2004). Thus, these starch synthesis-related genes (SSRGs) naturally have great influence on the synthesis of RS. A high-RS mutant has been developed by influencing the expression of starch branching enzyme 3 (SBE3) (Yang et al. 2012). Zhou et al. (2016) believed that soluble starch synthasellla (SSIIla) can regulate RS content and further revealed that RS yield is dependent on the high expression of $W x^{a}$ allele, which is prevalent in indica varieties. Krishnan et al. (2019) analyzed the correlation between PUL activity and inherent RS (RS1, RS2, RS5) and found that high PUL activity contribute to inherent RS. In other cereals, the down- regulation of soluble starch synthasell-3 (SSIla) and SBE could increase RS content in barley (Topping et al. 2003; Bird et al. 2004) and wheat (Hazard et al. 2014; Ahmed et al. 2015). Due to the molecular basis regulating the RS synthesis is largely unclear, to identify the RS genes is crucial for clarifying the RS synthesis and for the breeding of high-RS varieties.

It is generally considered that eating and cooking qualities (ECQs) are the most crucial rice qualities, which are mainly comprised of three physicochemical properties: apparent amylose content (AAC), gel consistency (GC), and gelatinization temperature (GT) (Tian et al. 2009). In recent years, because of its advantages of simple and rapid, the rapid visco analyzer (RVA) has been widely employed to evaluate the rice ECQs. The AAC shows a significantly positive correlation with RS content (Kong et al. 2015). Most of rice varieties with eminent RS content tend to have high AAC and poor taste, which is not acceptable to most consumers. Therefore, it is an important goal to study the relationships between RS and 
physicochemical properties in rice, which can make a foundation for obtaining rice varieties with elevated RS content and good taste. In this study, the recombinant inbred lines (RILs) were employed to study the effects of $W x$ and SSIla on RS content, and the relationships between RS and physicochemical properties were analyzed, aiming to provide useful information for molecular breeding of rice RS.

\section{Materials And Methods}

\section{Plant Materials}

In present study, CG133R (Oryza sativa ssp. indica, a restorer line, $A A C \approx 27 \%$ ) and Javanica22 (AAC $\approx$ $0.7 \%$ ) were used to construct RIL. Javanica 22 was a natural variation which derived from Xiangdali. Glutinous identification showed that the second exon of their waxy gene had nucleotides insertions of 23 bp, namely, there was recessive $w x w x$ genotype for them (You et al. 2019). The alleles of two parents differed in ADPglucose pyrophorylase small unit (AGPsma), ADPglucose pyrophorylase large unit (AGPlar), Wx, SSIla, soluble starch synthasellIb (SSIIIb) and soluble starch synthaselV-2 (SSIV-2) (Fig. 1). At seventh generation, a total of 99 lines with homozygous genetic background of SSRGs were identified from 142 lines and then a single plant was harvested from each line and taken as experimental materials when they were mature. All the materials were grown in an experimental agricultural field of the Southwest University of Science and Technology (Sichuan, China) and maintained normal management.

Methods

\section{Determination of resistant starch and physicochemical properties}

Harvested rice grains were air-dried and stored at room temperature for 3 months to balance water before analyses. Rice was dehulled by using a Dehuller (TR-200; Kett, Tokyo, Japan). The brown rice was polished by using polisher (Pearlest; kett, Tokyo, Japan) and subsequently ground to flour in a Laboratory Mill (LM3100; Petern, Malmö, Sweden) with a 100 mesh sieve for measuring RS content and physicochemical properties.

Cooked rice was prepared according to the Zhou's method (Zhou et al. 2016) and taken a part of cooked rice stored at $4{ }^{\circ} \mathrm{C}$ for a week to product retrograded rice. In this work, RS content was measured in three sample types, including raw milled rice, cooked rice and retrograded rice by using RS Rapid Assay Kit (Megazyme, Bray, Co. Wicklow, Ireland), according to the manufacture's assay procedure. Each starch sample was measured in triplicate.

AAC and GC were measured according to the standard of Chinese Ministry of Agriculture, NY/T 26392014, and Chinese national standards, GB/T 17891-1999, respectively. GT was estimated by differential scanning calorimetry (DSC) according to the methods reported previously (Yan et al. 2010). In order to measure sample percentage of retrogradation (R\%), the gelatinized starch was stored at $4^{\circ} \mathrm{C}$ for 7 days. Afterwards, it was equilibrated to room temperature for $1 \mathrm{~h}$ and then with the same thermal program as the measurement of GT. R\% was calculated by the following formula: 
$\mathrm{R} \%=\Delta \mathrm{Hr}$ (enthalpy of retrogradation) $/ \Delta \mathrm{Hg}$ (enthalpy of gelatinization)

Each index of per sample was measured in duplicate.

\section{Measurement of RVA profile characteristics}

A RVA (RVA4500, NewPortSci. Co. Warriewood, Australia) was used to estimate RVA profile characteristics in accordance with the American Association of Cereal Chemists (AACC) operational procedure. Three original parameters obtained from the RVA profile as following: peak viscosity (PKV), hot paste viscosity (HPV), cool paste viscosity (CPV); and three secondary parameters including: breakdown viscosity (BDV=PKV-HPV), setback viscosity (SBV=CPV-PKV), consistence viscosity $(\mathrm{CSV}=\mathrm{CPV}-\mathrm{HPV})$. Besides that, the pasting temperature $(\mathrm{PaT})$ and pasting time (PeT) also were recorded. Each starch sample was measured in duplicate.

\section{Analysis of starch granule shape and particle size distribution}

Rice starch granules were isolated according to the procedures of peng et al. (1999). The shape of starch granule was observed using scanning electron microscopy (SEM)H, Oberkochen, . Particle size distribution of starch was determined with a laser diffraction particle size analyser (Model LS 13320, Beckman Coulter, USA). Granule size was represented with mean diameter automatically calculated by the instrument software.

\section{DNA extraction and genotyping}

About $100 \mathrm{mg}$ fresh young leaf was sampled from each plant and grounded using a Fastprep Sample Rapid Crushing System (MP Biomedicals, Santa Ana, CA, USA). The genomic DNA was extracted with the CTAB method (Murray and Thompson 1980).

A total of $10 \mu \mathrm{L}$ reaction mixtures were employed to identify the genotypes of 18 SSRGs alleles between two parents by using the Golden Easy PCR System (TIANGEN, Beijing, China) and the primers designed by Tian et al. (2010); Cai et al. (2002) and Zhou et al. (2016) (Additional file 2: Table S1). PCR-ACC/was designed to detect the first intron $\mathrm{G} / \mathrm{T}$ polymorphism of $W x$ and $W x \mathrm{M} 1$ was used to distinguish glutinous rice varieties from the others. And then, the molecular markers of genes with polymorphism between two parents were selected to detect the genotypes of 99 lines. All the primers were synthesized by Sangon Biotech (Shanghai, China). Polymerase chain reactions (PCR) ran on an Eppendorf Thermal Cycler (Mastercycler ${ }^{\circledR}$ nexus GSX1, Germany). $5 \mu \mathrm{L}$ of each amplification products of CAPS primers was digested with corresponding restriction endonuclease in a total of $15 \mu \mathrm{L}$ enzymatic system including 1.5 $\mu \mathrm{L} 10 \times$ buffer, $8 \mu \mathrm{L} \mathrm{ddH_{2 }} \mathrm{O}$ and $0.5 \mu \mathrm{L}$ restriction endonuclease. The digestion reactions were performed by using an Eppendorf Thermal Cycler at $37^{\circ} \mathrm{C}$ for $3-4 \mathrm{~h}$. All the amplified products were detected on a $3 \%$ agarose gel in $0.5 \times$ Tris-Borate EDTA (TBE) buffer using GreenView (Applied BioProbes, Rockville, MD, USA).

\section{Statistical analyses}


Analysis of variance (ANOVA) and multiple comparisons of the Duncan method were performed with the Statistical Product and Service Solutions software version 22 (SPSS, https://www.ibm.com/analytics/cn/zh/technology/spss/) after the data being classified into groups according to the genotyping results. In addition, the correlation analysis between RS content and physicochemical properties was conducted by using SPSS 22 .

\section{Results}

\section{Analysis on genetic background of materials and grouping of RIL}

Polymorphism of eighteen SSRGs had been detected between two parents and they differed in 6 genes (AGPsma, AGPlar, SSIIIb, SSIV-2, Wx and SSIIa). Genetic analysis on SSRGs of the 99 lines came from the RIL showed that there were different allelic genotypes for the 6 genes (Fig. 1). Among the experimental population, statistically significant differences on RS were only detected in different $W x$ alleles and SSIla alleles $(P<0.05)$ (Additional file 2: Table S2). So we only focused on the effects of $W x$ and SSIla alleles for RS content. $W x$ genotypes of the lines were identified by utilizing molecular marker PCR-AcCl and results indicated that the lines could divided into two groups: $W x^{a} W x^{a}$ genotype and $W x^{b} W x^{b}$ genotype. And then, glutinous identification showed that all lines with the background of $W x^{b}$ were glutinous rice, which were recessive $w x w x$ genotype (Fig. 1, Additional file 1: Fig. S1). Because SSIla alleles amplified by SSII-3 M1 were haplotype 1 (G/G/GC, indica-type) and 4 (A/G/TT, japonica-type) (You et al. 2020), the detected result by SSII-3 M1 showed that haplotype of SSIla of Javanica 22 and CG133R belonged to haplotype 1 and haplotype 4, separately (Fig. 1). Diverse groups were obtained according to different alleles among the 99 lines, and were designated as follows: to $W x$ alleles: $W x^{a}$ type (came from CG133R), $w x$ type (came from Javanica 22), respectively; to SSIla locus: SSIla-I type (same as Javanica 22) and SSIla-Il type (same as CG133R), respectively.

\section{Phenotypic variability of RS content in rice RIL and their parents}

A t-test was implemented in order to compare the difference of parents on RS content. The result showed that the parents differed significantly from each other on the RS content in raw milled samples (RSm), the RS content in cooked rice (RSc) and the RS content in retrograded rice (RSr) $(P<0.05)($ Table 1). RS content in three sample types showed a wide variations among the RIL, especially RSm, with an average of $10.61 \%$ and ranging from $0.03 \%$ to $29.42 \%$ (Table 1 ). Compared with the RSm, the average of RSc and RSr decreased by $9.88 \%$ and $9.55 \%$, respectively. The difference of RS content between raw milled samples and cooked rice was significant in RIL $(P<0.01)$, but there was no statistical difference on RS content between cooked rice and retrograded rice at 0.05 level (Additional file 1: Fig. S2). This fact indicated that RS contents of the different lines were decreased sharply after cooking, but no distinct variation was observed in RS content after retrogradated process. Therefore, the subtype of RS in the RIL was mainly RS2, and less RS3.

Table 1 Distribution of three types of RS in RIL and their Parents. 


\begin{tabular}{|lllll|}
\hline & RIL & & Javanica 22 & CG133R \\
\cline { 2 - 5 } Types & Rang & Mean \pm SD & Mean \pm SD & Mean \pm SD \\
\hline RSm(\%) & $0.03-29.42$ & $10.61 \pm 5.48$ & $2.12 \pm 0.05^{\star}$ & $5.32 \pm 0.15$ \\
\hline RSc(\%) & $0.00-1.71$ & $0.73 \pm 0.39$ & $0.01 \pm 0.01^{*}$ & $1.21 \pm 0.04$ \\
\hline RSr(\%) & $0.00-2.21$ & $1.06 \pm 0.50$ & $0.2 \pm 0.00^{*}$ & $1.96 \pm 0.13$ \\
\hline
\end{tabular}

RSm, resistant starch content in raw milled sample; RSc, resistant starch content in cooked rice; RSc, resistant starch content in retrograded rice. * indicates $\mathrm{P}<0.05$, according to Student's $t$-test.

\section{Correlation analysis between RS and physicochemical properties in RIL}

In this study, the physicochemical properties of the RIL and two parents were tested, which showed a wide variations among the tested materials (Table 2). The correlation analyses between physicochemical properties and RSm, RSC and RSr showed that the AAC, all DSC thermal parameters, PKV, PaT and CSV had a positive correlation with the RSm (Table 3). The AAC and all RVA profile parameters, except for PKV, BDV and PaT, were significantly positively correlated with RSc, while all DSC thermal parameters showed significantly negative correlation with RSc $(P<0.05)$, and the same result also showed in RSr. Besides that, all the correlation coefficients of RSr were lower than that of RSc, except for PKV. These data suggested that RSm, RSc and RSr would increase along with the rise of AAC, and higher RSm along with higher GT. However, RSc and RSr would decrease when GT increased.

Table 2 Physicochemical indexes of RIL and their parents. 


\begin{tabular}{|lllll|}
\hline Index of ECQs & RIL & & Javanica 22 & CG133R \\
\cline { 2 - 5 } & Rang & Mean \pm SD & Mean \pm SD & Mean \pm SD \\
\hline AAC & $0-26.09$ & $18.94 \pm 7.59$ & $0.68 \pm 0.00$ & $26.98 \pm 0.00$ \\
\hline GC & $1.80-11.55$ & $6.36 \pm 3.03$ & $11.3 \pm 0.10$ & $7.9 \pm 0.20$ \\
\hline T0 & $60.01-78.69$ & $67.54 \pm 5.70$ & $78.06 \pm 0.20$ & $62.08 \pm 0.12$ \\
\hline TP & $65.2-81.62$ & $71.91 \pm 5.01$ & $81.44 \pm 0.03$ & $67.09 \pm 0.08$ \\
\hline TC & $71.54-85.90$ & $77.32 \pm 4.42$ & $85.87 \pm 0.92$ & $72.69 \pm 0.34$ \\
\hline R\% & $12.03-53.9$ & $34.33 \pm 9.73$ & $46.64 \pm 0.00$ & $26.35 \pm 0.00$ \\
\hline PKV & $2683.5-4739$ & $3663.63 \pm 366.65$ & $3551 \pm 99$ & $3321 \pm 105$ \\
\hline HPV & $1053-3824$ & $2463.12 \pm 561.60$ & $1736 \pm 60$ & $2474 \pm 64$ \\
\hline CPV & $1339-5826$ & $4249.11 \pm 1098.05$ & $2224 \pm 78$ & $4674 \pm 100$ \\
\hline PeT & $3.53-6.33$ & $5.82 \pm 0.78$ & $4.4 \pm 0.00$ & $6.23 \pm 0.03$ \\
\hline PaT & $71.7-83.95$ & $76.52 \pm 3.80$ & $83.15 \pm 0.01$ & $72.48 \pm 0.03$ \\
\hline BDV & $492-2786$ & $1200.51 \pm 488.97$ & $1815 \pm 55$ & $847 \pm 41$ \\
\hline SBV & $-2022.5-2032$ & $585.48 \pm 988.82$ & $-1327 \pm 35$ & $1353 \pm 5$ \\
\hline CSV & $286-3032$ & $1785.99 \pm 640.65$ & $488 \pm 10$ & $2200 \pm 36$ \\
\hline
\end{tabular}

AAC, apparent amylose content; GC, gel consistency; T0, gelatinization start temperature; TP, gelatinization peak temperature; Tc, gelatinization end temperature; $R \%$, percent of retrogradation; $P K V$, peak viscosity; HPV, hot paste viscosity; CPV, cool paste viscosity; PeT, peak time; PaT, pasting temperature; BDV, breakdown value; SBV, consistence value; SCV, setback value.

Table 3 Correlations between RS and Physicochemical Properties in RIL 


\begin{tabular}{|c|c|c|c|}
\hline Parameter & $\mathrm{RSm}$ & RSc & $\mathrm{RSr}$ \\
\hline AAC & $0.43^{* *}$ & $0.849 * \star$ & $0.828^{* *}$ \\
\hline $\mathrm{GC}$ & 0.096 & $-0.472^{\star \star}$ & $-0.432^{\star *}$ \\
\hline To & $0.244^{* *}$ & $-0.362^{\star \star}$ & -0.351 ** \\
\hline $\mathrm{Tp}$ & $0.56^{* *}$ & $-0.53^{\star \star}$ & $-0.486^{\star *}$ \\
\hline Tc & $0.481 * *$ & $-0.574^{\star \star}$ & $-0.542^{\star \star}$ \\
\hline$\Delta \mathrm{H}$ & $0.236^{*}$ & $-0.695^{\star \star}$ & $-0.667 * \star$ \\
\hline $\mathrm{R} \%$ & $0.531^{* *}$ & $-0.219 *$ & $-0.204^{\star}$ \\
\hline PKV & $0.288 * *$ & 0.084 & 0.092 \\
\hline HPV & 0.095 & $0.641^{\star \star}$ & $0.607 * *$ \\
\hline CPV & 0.18 & $0.802^{\star \star}$ & $0.781^{* *}$ \\
\hline РeT & 0.194 & $0.724^{\star \star}$ & $0.69 * \star$ \\
\hline РаT & $0.582^{\star *}$ & -0.408 *夫 & $-0.388^{\star \star}$ \\
\hline BDV & 0.106 & $-0.672^{\star \star}$ & -0.626 ** \\
\hline SBV & 0.094 & $0.857^{\star \star}$ & $0.83^{\star \star}$ \\
\hline CSV & $0.225^{\star}$ & $0.815^{\star \star}$ & $0.807 * *$ \\
\hline
\end{tabular}

$\mathrm{RSm}$, resistant starch content in raw milled sample; RSc, resistant starch content in cooked rice; RSr, resistant starch content in retrogradation; AAC, apparent amylose content; GC, gel consistency; To, gelatinization start temperature; Tp, gelatinization peak temperature; Tc, gelatinization end temperature; $\Delta \mathrm{H}$, Enthalpy change; $\mathrm{R} \%$, percent of retrogradation; PKV, peak viscosity; HPV, hot paste viscosity; CPV, cool paste viscosity; $\mathrm{PeT}$, peak time; $\mathrm{PaT}$, pasting temperature; $\mathrm{BDV}$, breakdown value; $\mathrm{SBV}$, consistence value; CSV, setback value. ${ }^{\star \star *}$ correlations are significant at $p<0.05, p<0.01$, respectively.

\section{Effects of $W x$ alleles on RS in the RIL}

Among the 99 experimental lines, $W x$ alleles were divided two groups: $W x^{a}$ type and $w x$ type. Effects of $W x$ alleles on rice RS were investigated by ANOVA. The result demonstrated that there were significant differences on RSm, RSc and RSr under different $W x$ alleles $(\mathrm{P}<0.01)$ (Fig. 2a). The average of RSm, RSc and $\mathrm{RSr}$ of $W x^{a}$ type increased by $9.54 \%, 0.85 \%$ and $1.21 \%$, respectively, compared with that of $w x$ type. The result suggested that $W x^{a}$ could improve RS content in three sample types.

Furthermore, the differences between RSm and RSc, RSr and RSc were calculated. RSa= RSm - RSc, RSb= $\mathrm{RSr}$ - RSc. RSa mainly was RS2 and RSb mainly was RS3. Result of ANOVA revealed that RSa varied significantly under different alleles of $W x$, as did RSb $(P<0.01)$ (Fig. 2b). RSa and RSb of $w x$ type $(2.53 \%$ 
and $0.05 \%$, respectively) also were extremely significantly lower than that of $W x^{a}$ type $(11.21 \%$ and $0.41 \%)$ $(P<0.01)$. This result indicated that $W x$ alleles could affect RS2 and RS3 and $W x^{a}$ could promote RS2 and RS3 content.

In order to further confirm the above results, a sub-population with total of 16 lines, which only varied in $W x$ locus under the genetic background of CG133R for SSRGs, were selected from 99 lines, of which 6 lines belonged to $w x$ type and 10 lines belonged to $W x^{a}$ type. Statistical differences were detected in RSm, RSc, RSr, RSa and RSb under different $W x$ alleles by ANOVA, respectively. The result of $t$-test indicated that the average of RS content of $w x$ type significantly differed from $W x^{a}$ type $(P<0.01)$. The means of RSm, RSc, RSr, RSa and RSb of $W x^{a}$ type increased by $4.89 \%, 1.00 \%, 1.45 \%, 3.88 \%$ and $1.45 \%$, respectively, compared with that of $w x$ type (Fig. $2 \mathrm{c}$ and d). It could be furtherly confirmed that $W x$ had a great influence on rice RS content that could be increased by $W x^{a}$.

\section{Effects of SSIla alleles on RS in the RIL}

Ninety-nine lines were divided into two groups by SSIla alleles: SSIla-I type and SSIla-Il type, and their RS content distinctly differed from each other in the three sample types $(P<0.01)$ (Fig. 3a). RSm of SSIla-I type was $17.83 \%$, and that of SSIla-ll type only $5.57 \%$. However, the RSc and RSr of SSIla-l type were lower than that of SSIla-ll type. It showed that SS/la allelic polymorphism had an important impact on rice RS, but the specific role needed further confirmation. Moreover, the difference of RSa was significant under different SSIla alleles by ANOVA ( $P<0.01)$ (Fig. 3b). The RSa of SSIla-l type was $17.27 \%$, which also differed from SSIla-l type (4.74\%). In fact, SSlla played an important role in controlling rice RS, except for RS3.

Furthermore, 18 lines that only had polymorphisms in SSIla locus were selected from RIL under the CG133R genetic background for SSRGs, of which 6 lines belonged to SSIla-l type and 12 lines belonged to SSIla-l type. Significant differences were detected in RSm or RSa under different SSIla alleles by $t$ test. RSm of SSIla-l type (21.33\%) was obviously higher than that of SSlla-l type (5.56\%) (Fig. 3c). RSa of SSIla-I type (20.44\%) was significantly higher than that of SSIla-ll type $(4.52 \%)(P<0.01)$ (Fig. 3d). The result indicated that SSIla had important effect on rice RS2.

\section{Combined effects of $W x$ and SSIla alleles on RS}

A total of 24 lines, which only separated in Wx and SSIla loci under the background of CG133R for SSRGs in the 99 lines, were isolated to analyze their combined effects on RS (Additional file 2: Table S3). Results demonstrated that the interacting effect of $W x$ and SSIla alleles for RSc was significant $(P<0.05)$, and that for RSm, RSr and RSa were significant $(P<0.01)$ (Additional file 2: Table S4). The combined effects of $W x$ and SSIla alleles on rice RS must be further studied.

There were four combinations among the tested rice lines: $w x-I, w x-I I, W x^{a}-I$ and $W x^{a}-I I$, which came from the combination of $W x$ and SSIla alleles (Fig. 4). RSm of $w x-I$ (7.79\%) was similar to that of $W x^{a}-I I$ 
(5.59\%), which was significantly higher than that of $w x-I I(0.67 \%)$, but distinctly lower than that of $W x^{a}-I$ (21.33\%) $(P<0.01)$, and RSa with same effect. The significant differences on RSm and RSa between homozygotes of $W x^{a}$ and $w x$ were detected under the same background of SSIla alleles. In addition, there were statistical differences on RSm and RSa between homozygotes of different SSlla alleles under the same background of $W x$. RSc of $W x^{a}-I(0.89 \%)$ and $W x^{a}-I I(1.04 \%)$ was significantly higher than that of $w x-I(0.06 \%)$ and $w x-I I(0.04 \%)$. Likewise, the effects of $W x$ alleles on RSc were significant different under the same SSIla alleles. However, the differences on RSc between homozygotes of different SSIla alleles were not significant under the same background of $W x$. And the same result also showed in RSr. These results indicated that $W x$ had an important influence on RS under the same background of SSIla alleles, and SSIla only played a significant role in regulating RSm and RSa under the same background of $W x$ alleles.

\section{Characterization of starch granule shape and particle size distribution in different $W x$ and SSIla allelic combinations}

A single plant that were selected from each combinations ( $w x-I, w x-I I, W x^{a}-I$ and $\left.W x^{a}-I I\right)$, respectively, was used to determine its starch granule shape and particle size distribution (Additional file 2: Table S5). Figures of scanning electron microscopy revealed that starch granules of the single plant with different genotypes were variable in shape and size. And most of starch granules in tested plants were polygonal and a few were rounded (Fig. 5). Results of starch granule distribution demonstrated that particle size had a wide variations in the samples with different genotypes, and most of them belonged to medium size starch granules $(4 \mu \mathrm{m} \leq$ granular diameter $\leq 8 \mu \mathrm{m})$ (Table 4). Moreover, there were statistical differences in particle size distribution among different combinations. The combination $W x^{a}$-II had the lowest proportion of medium size starch granules and the highest proportion of larger size starch granules (granular diameter $>8 \mu \mathrm{m}$ ), compared with the other three combinations. And the mean diameter of $W x^{a}-I I(7.56 \mu \mathrm{m})$ was significantly higher than that of other three combinations $(P<0.05)$. In addition, effects of $W x$ and SSIla alleles on starch granule distribution were further analyze by a split block design (Additional file 2: Table S6). The results indicated $W x$ and SSIla alleles had an effect on particle size of starch, especially SSIla alleles could significantly affect the volume of starch granules of different sizes $(P<0.01)$. Furthermore, a correlation analysis was implemented in order to investigate the relationships between starch granule volume and RS content, and the result showed that it was not correlated with granule volume to the RSm, RSc, RSr, RSa and RSb (Additional file 2: Table S7). To sum up, particle size of the four combinations of $W x$ and SSIla alleles were different, but there was no correlation between RS content and starch granule volume.

\section{Table 4 Difference in particle size distribution of starch granule among different combinations of $W x$ and SSIla}




\begin{tabular}{|c|c|c|c|c|c|}
\hline \multirow{3}{*}{ Genotype } & \multirow{3}{*}{$\begin{array}{l}\text { Mean diameter } \\
(\mu \mathrm{m})\end{array}$} & \multicolumn{4}{|c|}{ Starch granule distribution } \\
\hline & & \multicolumn{4}{|c|}{ Volume percentage (\%) } \\
\hline & & $0-2 \mu \mathrm{m}$ & $2-4 \mu \mathrm{m}$ & $4-8 \mu \mathrm{m}$ & $>8 \mu \mathrm{m}$ \\
\hline$w x-1$ & $5.11 \pm 0.02 b$ & $17.74 \pm 0.13 b B$ & $24.03 \pm 0.04 \mathrm{bB}$ & $41.55 \pm 0.09 \mathrm{bA}$ & $16.68 \pm 0.18 \mathrm{bB}$ \\
\hline$w x-I I$ & $5.65 \pm 0.04 b$ & $15.29 \pm 0.14 \mathrm{dC}$ & $26.77 \pm 0.15 \mathrm{aA}$ & $42.36 \pm 0.41 \mathrm{abA}$ & $15.58 \pm 0.42 b B$ \\
\hline$W x^{a}-1$ & $5.67 \pm 1.19 b$ & $19.85 \pm 0.51 \mathrm{aA}$ & $24.26 \pm 0.60 \mathrm{bB}$ & $43.45 \pm 1.13 a \mathrm{~A}$ & $12.43 \pm 2.23 \mathrm{cB}$ \\
\hline$W x^{a}-11$ & $7.56 \pm 0.04 a$ & $16.3 \pm 0.12 \mathrm{cC}$ & $16.64 \pm 0.07 c C$ & $39.22 \pm 0.05 \mathrm{cB}$ & $27.84 \pm 0.09 a A$ \\
\hline
\end{tabular}

I, II Type indicate homozygote for Janavica 22 and CG133R in SSIla locus, respectively. Lowercase and capital letters above column denote significant at 0.05 and 0.01 levels, respectively.

\section{Discussion}

\section{Types of resistant starch in the rice RIL}

RS2 and RS3 are two main subtypes of RS, which are resistant to enzymatic hydrolysis. RS2 consists of native starch granules. It is reported that RS2 has a unique crystalline structure with B-type X-ray diffraction pattern, which is resistant to the enzymatic digestions (Topping and Clifton 2001). However, its resistance to enzymatic treatment will greatly decrease after hydrothermal processing (Wang and Copeland 2013). Chung et al. (2006) found that it was more susceptible to hydrolysis for RS2 after heat process or cooking, and most of RS2 in the native waxy starch will be transformed into enzymatic starch after cooking. In this study, the average of RS content in raw starch samples was $10.61 \%$, but its RS content dramatically decreased after cooking (Table 1). We deduced that the subtype of RS in these materials was mainly RS2 according to the characteristics of RS2. RS3 is retrograded starch, mainly coming from the gelatinized starch after cooling (Sharma et al. 2008), thermally very stable and hardly digested. RS content in these plant materials increased by $0.33 \%$ after storage at $4{ }^{\circ} \mathrm{C}$ for 7 days, but the difference was not significant between RSc (0.73\%) and RSr (1.06\%) $(P<0.05)$ (Table 1, Additional file 1: Fig. S2). This result revealed that RS3 in these materials was low. Briefly, the subtype of RS was mainly RS2 in this RILs, while RS3 was less.

\section{Relationships between RS andrice quality indices}

AAC and amylopectin fine structure of rice starch also determines RS content in addition to affecting the physicochemical properties. Therefore, it is necessary to analyze the relationship between RS content and physicochemical properties of rice. Zhang et al. (2007) and Shu et al. (2014) revealed that AAC was positively correlated with RS. Furthermore, Ding et al. (2019) found that the effect of AAC on RS in raw rice was not as great as that on RS in cooked rice, which is consistent with our result and the correlation coefficient between AAC and RSm was smaller than that between AAC and RSc $\left(R^{2}=0.43,0.849\right.$, 
respectively, $\mathrm{P}<0.01$; Table 3$)$. The correlation of RVA parameters and RSm were different from that and RSc. The former had a positive correlation at PKV, PaT and CSV, but the later with significantly positive correlation for most of RVA profile parameters. The results were similar to the previous result of correlation analysis reported by Ding et al. (2019). Furthermore, Chen et al. (2017) analyzed the correlation between RS and physiochemical properties of cooked rice and results showed that RS was negatively correlated with PKV and BDV. Nevertheless, Kong et al. (2015) indicated that RS had no correlation with PKV and BDV. Nakamura et al. (2015) found that RS was positive correlation with PKV and BDV. In our study, a significantly negative correlation between RS and BDV was detected $\left(R^{2}=-0.672\right.$, $P<0.01$; Table 3), but the relationship between RS and PKV was not observed. Moreover, it was reported that lower GT correlated with higher starch digestibility in vitro although their relationship was not statistically significant (Dhital et al. 2015). However, Yang et al. (2006) found that mutant RS111 with high RS content showed notably lower To, Tp, Tc and $\Delta \mathrm{H}$ values, compared with those of wild type and common rice. In present study, the gelatinized parameters such as To, Tp, Tc, $R \%$ and $\Delta H$ were negatively correlated with RSc, but positively correlated with RSm $(P<0.01)$.

\section{Implications of Wx and SSIla alleles on RS content in different process status}

It is accepted that $W x$ is the major gene controlling the amylose synthesis of rice. Previous studies indicated that $W x$ could influence the digestibility of starch and it was the major gene controlling Glycemic Index (GI) (Raben et al. 1994; Fitzgerald et al. 2011). Kong et al. (2015) made association test with 14 rice accessions to survey relationship between genetic and functional properties of rice starch and found that $W x$ could control the amount of RS. In this work, the results of genetic analysis demonstrated that $W x$ had a great effect on RS content and the $W x$ from CG133R $\left(W x^{a}\right)$ could improve RS content in rice (Fig. 2). $W x^{a}$ is one of the vital allele among the multiple alleles of $W x$, which produces a large amount of amylose (Itoh et al. 2003). Raigond et al. (2015) found that high amylose content could contribute to RS content. Bao et al. (2017) utilized the genome-wide association study to select the candidate genes affecting RS and identified that $W x$ was one of the candidate genes, as well as the mean RS content of indica was higher than that of japonica. Furthermore, Zhou et al. (2016) revealed that RS production was dependent on the high expression of $W x^{a}$ allele, which was consistence with our conclusion. It furtherly indicate that $W x$ affects RS through controlling amylose content.

SSIla plays a vital role in the amylopectin synthesis pathway, and it can control the degree of crystallinity (Kong et al. 2015). SSIla is present in a large complex including ADP-glucose pyrophosphorylase (AGPase), pyruvate orthophosphate dikinase (PPDK), SSIlla, SBElla and SBEllb, which regulate the partitioning of ADP-glucose (ADPGIC) into lipid or starch and further control RS5 (Zhou et al. 2016). Parween et al. (2020) found that the SNPs in exon 8 of SSIla played an important role in the formation of RS. However, Itoh et al. (2017) employed 7 rice lines to survey the pleiotropic effects of biosynthetic enzymes and indicated that SSIla could change the distribution of amylopectin chain but may not affect RS level. Our study showed that SSIla had an influence on rice RS except for RS3 in RIL (Fig. 3a and b), but only affected RS content in raw starch and RS2 in 18 lines (Fig. 3c and d). The RSm and RSa of 18 lines with SSIla allele from Javanica 22 (21.33\% and 20.44\%, respectively) were significantly higher than 
that from CG133R (5.56\% and $4.52 \%$, respectively) (Fig. 3c and d). The results of genetic analysis demonstrated that genotype of SSIla-I type (came from Javanica 22) belonged to indica SSIIa (SSIIai) and that of SSIla-II type (came from CG133R) belonged to japonica SSIla (SSIlaj) (Fig. 1). It is reported that SSlla played a critical role in elongating short A and B1 chain (DP 6-12) to long type B1 chains (DP 13-24) of amylopectin. And the activity of SSIla in indica rice was higher than that in japonica rice, which resulted in higher accumulation of B1 chains (Umemoto et al. 2002). Thus, it can be inferred that SSIla affects RS content in raw starch and RS2 by regulating the amount of intermediate-size chains of amylopectin.

\section{Combine effects of Wx and SSIla alleles on RS content and Phenotype of starch}

Multiple studies had shown that different combinations of Wx and SSIla genotypes have significant effects on the physicochemical properties of rice (Luo et al. 2015; Xiang et al. 2017). However, combined effects of $W x$ and SSIla alleles on RS content in rice have not been reported so far, the interaction of the two genes on RS were therefore analyzed. Apart from RS3, the combined effects of $W x$ and SSIla greatly affected RSm, RSc, RSr and RS2 (Additional file 2: Table S4). In the 24 lines, the RSm of $w x-1(7.79 \%)$ was similar to that of $W x^{a}-I I(5.59 \%)$, which was significantly higher than that of $w x-I I(0.67 \%)$, but distinctly lower than that of $W x^{a}-I(21.33 \%)(P<0.01)$, and RSa with same effect. The RSc and RSr of $W x^{a}-I(0.89 \%$ and $1.283 \%$, respectively) and $W x^{a}-I I(1.04 \%$ and $1.49 \%)$ were higher than that of $w x-1(0.06 \%$ and $0.15 \%)$ and $w x-I I(0.04 \%$ and $0.05 \%)$. There were significantly statistical differences on RS content in three sample types and RS2 between $W x^{a}$ and $w x$ alleles under the same SSIla allele, and the differences on RSm and RS2 between homozygotes of different SSIla alleles were significant under the same background of $W x$ (Fig. 4). These results suggested that $W x$ had a great impact on RSm, RSc, RSr and RS2 under the same SSIla alleles, and SSIla alleles only had effect on RSm and RS2 under the same Wx alleles.

It is generally considered that the granules in rice were polygonal starch granules with an irregularly shape and small size (3-8 $\mu \mathrm{m})$ (Patindol et al. 2007). Previous studies reported that the majority of starch granules from mutants with elevated RS were oval or rounded shape, such as RS111 (Yang et al. 2006), b10 (Zhou et al. 2016) and MR4 (Shu et al. 2014). Moreover, Shu et al. (2014) found that the size of starch granule of MR4 (high-RS mutant) was smaller than that of MR7 (low-RS mutant). But in the present study, the combinations of $W x$ and SSIla alleles with different RS content, whose starch granules mostly were polyhedral and sharp-edged (Fig. 5). There were statistical differences in particle size distribution among different combinations. $W x^{a}$-II had the lowest proportion of medium size starch granules and the highest proportion of larger size starch granules, compared with the other three combinations (Table 4). It implied that a high proportion of AAC and S-type amylopectin was in favor of the amyloplasta of the endosperm cells to forming large-diameter starch granules. Unfortunately, there was no correlation between RS content and starch granule diameter (Additional file 2: Table S7). Thus, we speculated that RS in the rice RIL may not be affected by starch phenotype, which regulated by $W x$ and SSIla alleles. Rice starch granule belongs to a kind of semi crystalline granule, which has a lamellar 
structure of alternating crystalline and amorphous layers. Within these lamellae, the crystalline layers are mainly composed of amylopectin chains, while the amorphous layers contain the amylopectin branching points and the disordered conformation of amylose and amylopectin molecules (Copeland et al. 2009). Li et al. (2019) pointed that the regulation of RS mainly focused on altering branch-chain lengths, amylose to amylopectin ratio, as well as the amorphous and crystalline structure of lamellae. The $W x$ is crucial for amylose synthesis, and the SSIla plays a vital role in the amylopectin synthesis. Therefore, $W x$ and SSIla alleles may manipulate the RS by controlling the crystalline structure of starch granules, which needs further verification. Based on our results and literature review, the genetic regulation of $W x$ and SSIla alleles on different type RS has a distinct pathway (Fig. 6).

In the present study, the subtype of RS in present study is mostly RS2 and less RS3, and RS content is closely relative with many indexes of physicochemical properties. Wx has a great effect on RS content and $W x^{a}$ can elevate RS content in different processing rice. The different SSIla alleles result in the variation of RS in raw rice and RS2 and the RSm and RS2 of SSIlai are significantly higher than that of SSIlaj. There is a significant effects of $W x$ alleles on RS content in three sample types and RS2 under the same SSIla alleles, and SSIla alleles only has effect on RSm and RS2 under the same $W x$ alleles. The combined effects of $W x$ and SSIla greatly affected RSm, RSc, RSr and RS2. The RSm of $w x-1$ was significantly higher than that of $w x-I I$, but distinctly lower than that of $W x^{a}-I(P<0.01) . W x^{a}-I I$ had the lowest proportion of medium size starch granules and the highest proportion of larger size starch granules compared with the other three combinations. Our discovery provides an effective way to further understand the molecular basis for genetic regulation of RS content, which may provide useful information for molecular breeding of rice RS.

\section{Abbreviations}

RS: resistant starch; SCFA: short chain fatty acids; ECQs: eating and cooking qualities; AAC: apparent amylose content; GC: gel consistency; GT: gelatinization temperature; RVA: rapid visco analyzer; RIL: recombinant inbred line; DSC: differential scanning calorimetry; R\%: percentage of retrogradation; PKV: peak viscosity; HPV: hot paste viscosity; CPV: cool paste viscosity; BDV: breakdown viscosity; SBV: setback viscosity; CSV: consistence viscosity; PaT: pasting temperature; PeT: pasting time; Gl: glycemic index.

\section{Declarations}

\section{Ethics Approval and Consent to Participate}

Not applicable

\section{Consent for Publication}

Not applicable 
Availability of Data and Materials

All data generated or analysed during this study are available within in this article or its supplementary files.

\section{Competing Interests}

The authors declare that they have no competing interests.

\section{Funding}

This work was supported by the Key Research \& Development program of Sichuan Province, China (Grant No. 2019YFN0019), the Key Program of Education Bureau of Sichuan Province, China (Grant No. 17ZA0412), and the Postgraduate Innovation Fund Project by Southwest University of Science and Technology (Grant No. 20ycx0038).

\section{Authors' Contributions}

$\mathrm{HY}$ and $\mathrm{LX}$ investigated genetic studies. $\mathrm{HY}, \mathrm{CL}$ and $\mathrm{OZ}$ carried out the measure of resistant starch and physicochemical properties. YC planted the materials and the pre-processing of samples. XX designed the overall project. $\mathrm{HY}$ and $\mathrm{OZ}$ analyzed the data and wrote the manuscript. All authors read and approved the final manuscript.

\section{Acknowledgements}

Not applicable

\section{References}

1. Ahmed R, Pierre B, Behjat KH, Suzhi L, Mark C, Oscar L, Anthony RB, Steve MS, Colin C, Stephen AJ, Zhong YL, Matthew M (2015) A genetic strategy generating wheat with very high amylose content. Plant Biotechnol J 13:1276-1286

2. Asp NG (1992) Preface resistant starch - Proceedings from the second plenary meeting of EURESTA European FLAIR Concerted Action no. 11 on physiological implications of the consumption of resistant starch in man. Eur J Clin Nutr 46:S1-148

3. Bao J, Zhou X, Xu F, He Q, Park YJ (2017) Genome-wide association study of the resistant starch content in rice grains. Starch - Stärke 69:1600343

4. Bird AR, Flory C, Davies DA, Usher S, Topping DL (2004) A novel barley cultivar (Himalaya 292) with a specific gene mutation in starch synthase lla raises large bowel starch and short-chain fatty acids in rats. J Nutr 134:831-835

5. Cai XL, Liu QQ, Tang SZ, Gu MH, Wang ZY (2002) Development of a molecular marker for screening the rice cultivars with intermediate amylose content in Oryza sativa subsp. indica. Acta 
photophysiologica sinica 28:137-144

6. Chen MH, Bergman CJ, McClung AM, Everette JD, Tabien RE (2017) Resistant starch: variation among high amylose rice varieties and its relationship with apparent amylose content, pasting properties and cooking methods. Food Chem 234:180-190

7. Chung HJ, Lim HS, Lim ST (2006) Effect of partial gelatinization and retrogradation on the enzymatic digestion of waxy rice starch. J Cereal Sci 43:353-359

8. Copeland L, Blazek J, Salman H, Tang MC (2009) Form and functionality of starch. Food Hydrocoll 23:1527-1534

9. Dhital S, Dabit L, Zhang B, Flanagan B, Shrestha AK (2015) In vitro digestibility and physicochemical properties of milled rice. Food Chem 172:757-765

10. Ding Y, Huang JJ, Zhang N, Rasmussen SK, Wu DX, Shu XL (2019) Physiochemical properties of rice with contrasting resistant starch content. J Cereal Sci 89:102815

11. Eggum BO, Juliano BO, Perez CM, Acedo EF (1993) The resistant starch, undigestible energy and undigestible protein contents of raw and cooked milled rice. J Cereal Sci 18:159-170

12. Fitzgerald MA, Rahman S, Resurreccion AP, Concepcion J, Daygon VD, Dipti SS, Kabir KA, Klingner B, Morell MK, Bird AR (2011) Identification of a major genetic determinant of glycaemic index in rice. Rice 4:66-74

13. Frei M, Siddhuraju P, Becker K (2003) Studies on the in vitro starch digestibility and the glycemic index of six different indigenous rice cultivars from the Philippines. Food Chem 83:395-402

14. Hazard B, Zhang X, Naemeh M, Dubcovsky J (2014) Registration of durum wheat germplasm lines with combined mutations in SBElla and SBEIlb genes conferring increased amylose and resistant starch. J Plant Regist 8:334-338

15. Hernández $\mathrm{O}$, Emaldi U, Tovar J (2008) In vitro digestibility of edible films from various starch sources. Carbohyd Polym 71:648-655

16. Hu PS, Zhao HJ, Duan ZY, Lin ZL, Wu DX (2004) Starch digestibility and the estimated glycemic score of different types of rice differing in amylose contents. J Cereal Sci 40:231-237

17. Itoh K, Ozaki H, Okada K, Hori H, Takeda Y, Mitsui T (2003) Introduction of $W x$ transgene into rice $w x$ mutants leads to both high- and low-amylose rice. Plant Cell Physiol 44:473-480

18. Itoh Y, Crofts N, Abe M, Hosaka Y, Fujita N (2017) Characterization of the endosperm starch and the pleiotropic effects of biosynthetic enzymes on their properties in novel mutant rice lines with high resistant starch and amylose content. Plant Sci 258:52-60

19. Kim JH, Tanhehco EJ, Ng PKW (2006) Effect of extrusion conditions on resistant starch formation from pastry wheat flour. Food Chem 99:718-723

20. Korus J, Witczak M, Ziobro R, Juszczak L (2009) The impact of resistant starch on characteristics of gluten-free dough and bread. Food Hydrocoll 25:988-995

21. Kong XL, Chen YL, Zhu P, Sui ZQ, Corke H, Bao JS (2015) On the relationships among genetic, structural and functional properties of rice starch. J Agr Food Chem 63:6241-6248 
22. Krishnan V, Awana M, Mahesh KS, Sunil IW, Ankur K, Ray M, Bollinedi H (2020) Pullulanase activity: A novel indicator of inherent resistant starch in rice ( Oryza sativa L.). Int J Biol Macromol 152:12131223

23. Li H, Gidley MJ, Dhital S (2019) High-Amylose Starches to Bridge the"Fiber Gap": Development, Structure, and Nutritional Functionality. Compr Rev Food Sci F 18:362-379

24. Luo J, Jobling SA, Millar A, Morell MK, Li Z (2015) Allelic effects on starch structure and properties of six starch biosynthetic genes in a rice recombinant inbred line population. Rice 8:15.

25. Murray MG, Thompson WF (1980) Rapid isolation of high molecular weight plant DNA. Nucleic Acids Res 8:4321-4326

26. Nakamura S, Katsura J, Kato K, Ohtsubo K (2015) Development of formulae for estimating amylose content and resistant starch content based on the pasting properties measured by RVA of Japonica polished rice and starch. Biosci Biotech Bioch 80:329-340

27. Parween S, Anonuevo JJ, Butardo Jr VM, Misra G, Anacleto R, Llorente C, Kosik O, Romero MV, Bandonill EH, Mendioro MS, Sreenivasulu N (2020) Balancing the double-edged sword effect of increased resistant starch content and its impact on rice texture: its genetics and molecular physiological mechanisms. Plant Biotechnol J 18:1763-1777

28. Patindol JA, Gonzalez BC, Wang YJ, McClung AM (2007) Starch fine structure and physicochemical properties of specialty rice for canning. J Cereal Sci 45:209-218

29. Peng MS, Gao M, Abdel-Aal ESM, Hucl P, Chibbar RN (1999) Separation and characterization of Atype and B-type starch granules in wheat endosperm. Cereal Chem 76:375-379

30. Raben A, Tagliabue A, Christensen NJ, Madsen J, Holst JJ, Astrup A (1994) Resistant starch: the effect on postprandial glycemia, hormonal response, and satiety. Am J Clin Nutr 60: 544-551

31. Raigond P, Ezekiel R, Raigond B (2015) Resistant starch in food: a review. J Sci Food Agr 95:19681978

32. Robertson JA, Monredon FD, Dysseler P, Guillon F, Amado R, Thibault JF (2000) Hydration properties of dietary fibre and resistant starch: a european collaborative study. LWT - Food Sci Technol 33:72-79

33. Sharma A, Yadav BS, Ritika (2008) Resistant starch: physiological roles and food applications. Food Rev Int 24:193-234

34. Shu XL, Sun J, Wu DX (2014) Effects of grain development on formation of resistant starch in rice. Food Chem 164:89-97

35. Tian ZX, Qian Q, Liu QQ, Yan MX, Liu XF, Yan CJ, Liu GF, Gao ZY, Tang SZ, Zeng DL, Wang Y.H, Yu JM, Gu MH, Li JY (2009) Allelic diversities in rice starch biosynthesis lead to a diverse array of rice eating and cooking qualities. Proc Natl Acad Sci U S A 106:21760-21765

36. Tian Z, Yan C, Qian Q, Yan S, Xie H, Wang F, Xu JF, Liu G, Wang YH, Liu QQ, Tang S, Li JY, Gu MH (2010) Development of gene-tagged molecular markers for starch synthesis-related genes in rice. Chinese Sci Bull 33:3768-3777 
37. Topping DL, Clifton PM (2001) Short-chain fatty acids and human colonic function: roles of resistant starch and nonstarch polysaccharides. Physiol Rev 81:1031-1064

38. Topping DL, Morell MK, King RA, Li Z, Bird AR, Noakes M (2003) Resistant starch and health Himalaya 292, a novel barley cultivar to deliver benefits to consumers. Starch - Stärke 55:539-545

39. Topping DL, Bajka BH, Bird AR, Clarke JM, Cobiac L, Conlon MA, Morell MK, Toden S (2008) Resistant starches as a vehicle for delivering health benefits to the human large bowel. Microb Ecol Health Dis 20;103-108

40. Umemoto T, Yano M, Satoh H, Shomura A, Nakamura Y (2002) Mapping of a gene responsible for the difference in amylopectin structure between japonica-type and indica-type rice varieties. Theor Appl Genet 104:1-8

41. Wang S, Copeland L (2013) Molecular disassembly of starch granules during gelatinization and its effect on starch digestibility: a review. Food Funct 4:1564-1580

42. Xia J, Zhu D, Wang R, Cui Y, Yan Y (2018) Crop resistant starch and genetic improvement: a review of recent advances. Theor Appl Genet 131:2495-2511

43. Xiang XC, Kang CF, Xu SJ, Yang BW (2017) Combined effects of Wx and SSlla haplotypes on rice starch physicochemical properties. J Sci Food Agric 97:1229-1234

44. Yamamori M, Kato M, Yui M, Kawasaki M (2006) Resistant starch and starch pasting properties of a starch synthase Ila-deficient wheat with apparent high amylose. Aust J Agr Res 57:531-535

45. Yan CJ, Fang YW, Li M, Peng JC, Liu QQ, Tang SZ, Gu MH (2010) Genetic effect of PUL allelic variation on rice cooking and eating qualities. Acta Agronomica Sinica 36:728-735

46. Yang CZ, Shu XL, Zhang LL, Wang XY, Zhao HJ, Ma CX, Wu DX (2006) Starch properties of mutant rice high in resistant starch. J Agr Food Chem 54:523-528

47. Yang RF, Sun CL, Bai JJ, Lou ZX, Shi B, Zhang JM, Yan WG, Piao ZZ (2012) A putative gene sbe3-rs for resistant starch mutated from SBE3 for starch branching enzyme in rice (Oryza sativa L.). Plos One 7:e43026

48. You H, Xiang XC, Yang BW, Li HC, Liang X, Long YT (2019) Physiological Characters and Genetic Characteristics of Heavy Panicle Type Variant, Javanica 22 (Oryza sativa ssp. javanica). Biocatal Agric Biotechnol 27:961-971

49. You H, Zhang OL, Xu L, Liang C, Xiang XC (2020) Effects of soluble starch synthase lla allelic Variation on rice grain quality with different Waxy backgrounds. J Sci Food Agric. doi: 10.1002/jsfa.10582

50. Zhang W, Bi J, Yan X, Wang H, Zhu C, Wang J, Wan J (2007) In vitro measurement of resistant starch of cooked milled rice and physico-chemical characteristics affecting its formation. Food Chem 105:462-468

51. Zhou H, Wang L, Liu G, Meng X, Jing Y, Shu X, Kong X, Sun J, Yu H, Smith SM, Wu D, Li J (2016) Critical roles of soluble starch synthase SSIlla and granule-bound starch synthase Waxy in synthesizing resistant starch in rice. Proc Natl Acad Sci U S A 113:12844-12849 


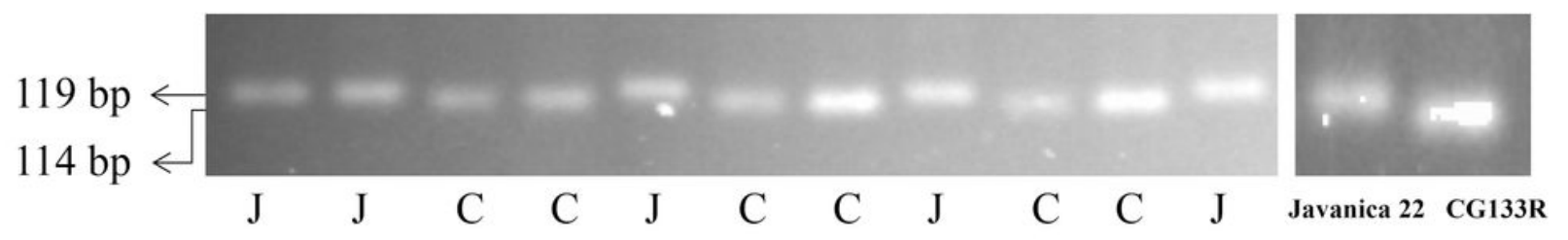

AGPlar M1

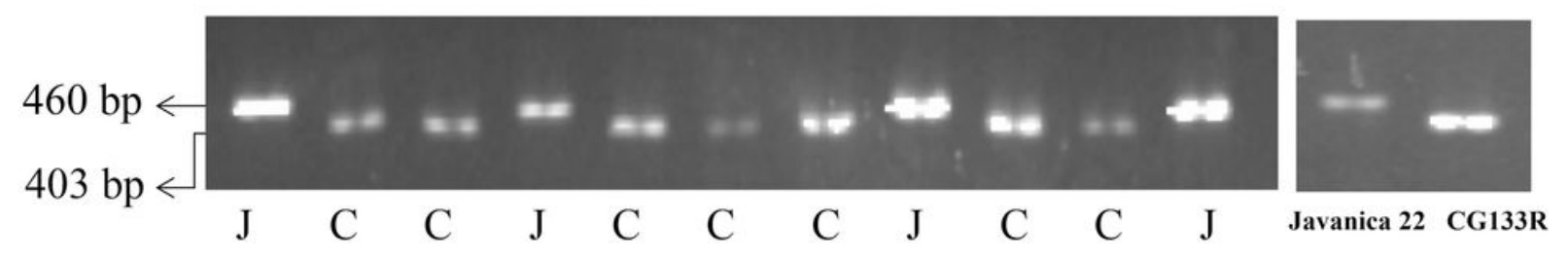

PCR-AccI
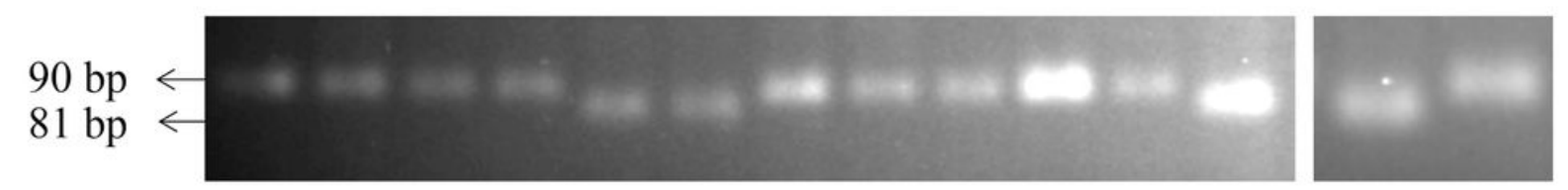

SSII-3 M1

$\begin{array}{llllllllllllll}\text { II } & \text { II } & \text { II } & \text { II } & \text { I } & \text { I } & \text { II } & \text { II } & \text { II } & \text { II } & \text { II } & \text { I } & \text { Javanica } 22 & \text { CG133R }\end{array}$

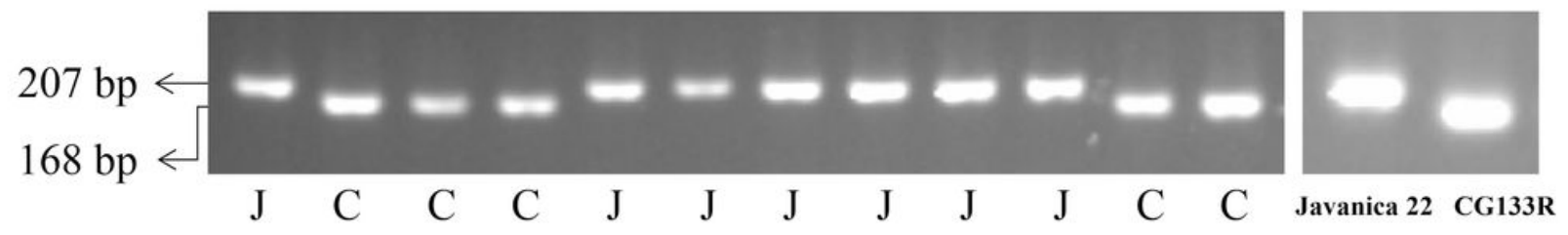

AGPsma 1

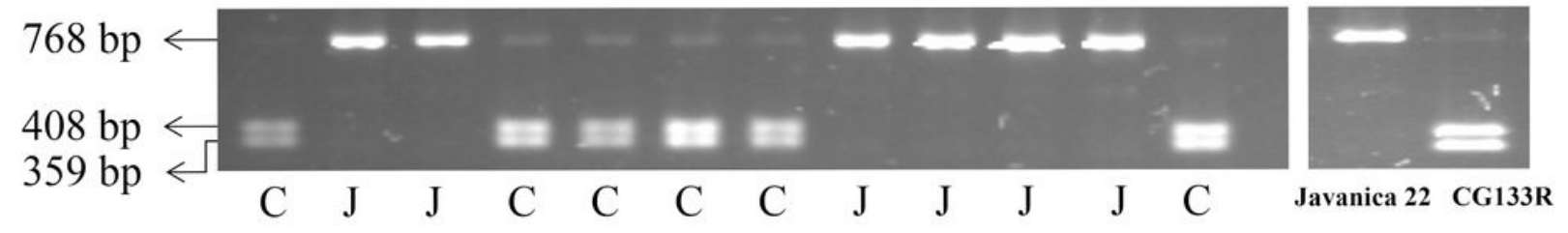

SSIII-1 t1

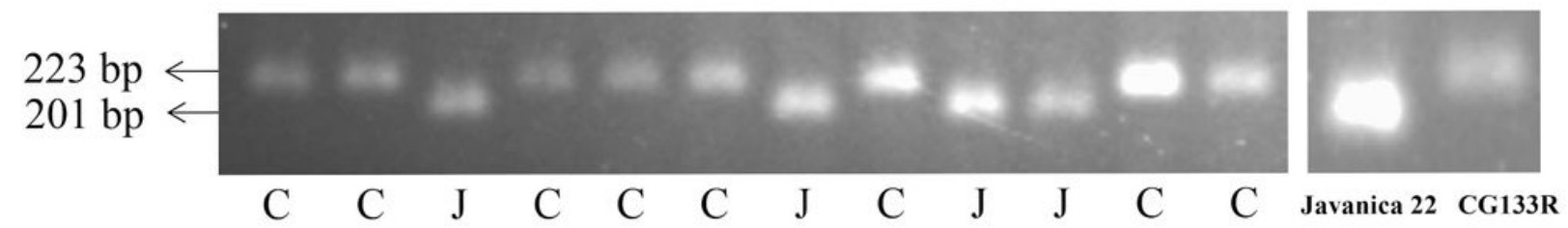

$S S I V-2 \mathrm{M} 2$

\section{Figure 1}

Polymorphism gene loci of starch synthesis-related genes between two parents and partial tested materials. J and I indicate genotype of Janavica 22, and C and II indicate genotype of CG133R. 


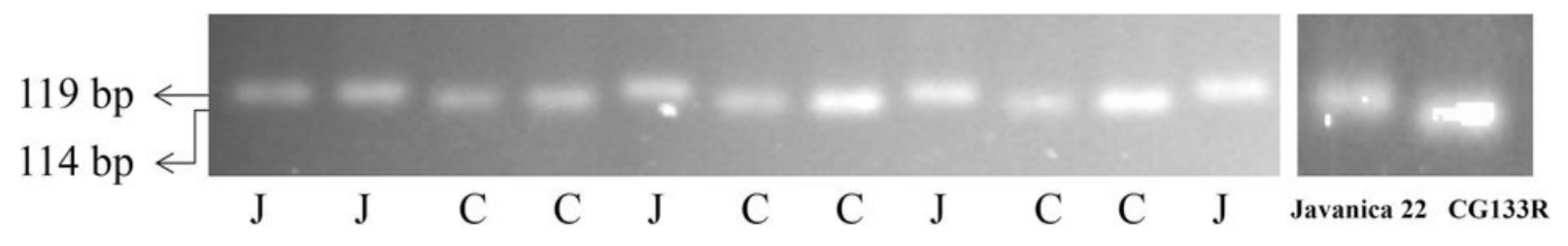

AGPlar M1

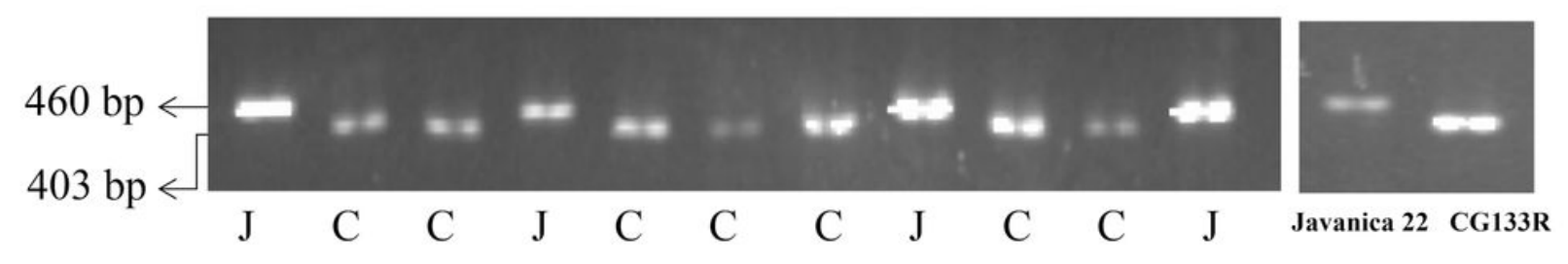

PCR-AccI

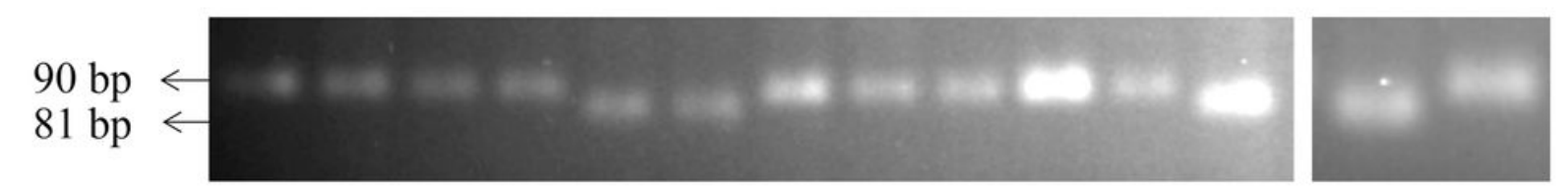

SSII-3 M1

$\begin{array}{llllllllllllll}\text { II } & \text { II } & \text { II } & \text { II } & \text { I } & \text { I } & \text { II } & \text { II } & \text { II } & \text { II } & \text { II } & \text { I } & \text { Javanica } 22 & \text { CG133R }\end{array}$

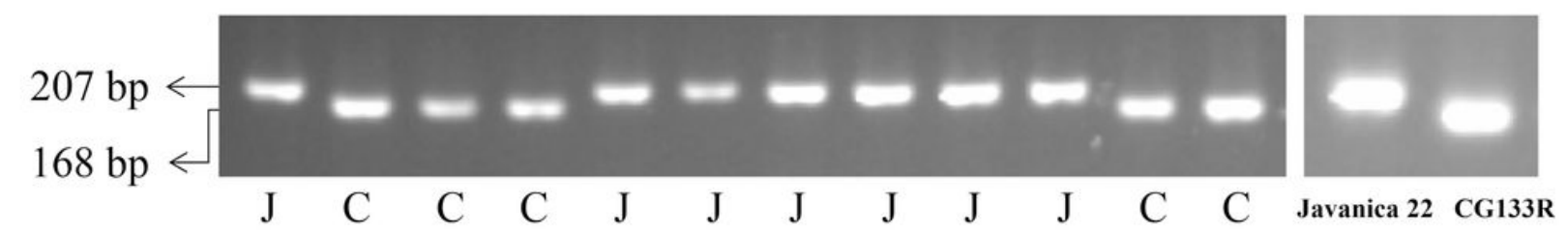

AGPsma 1

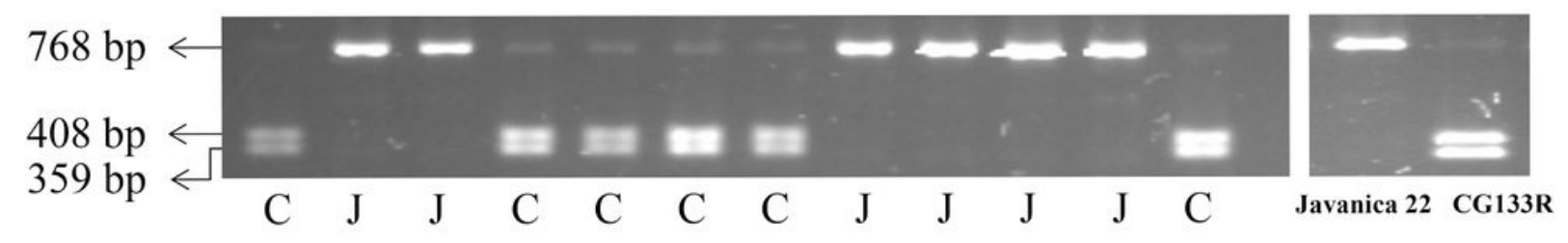

SSIII-1 t1

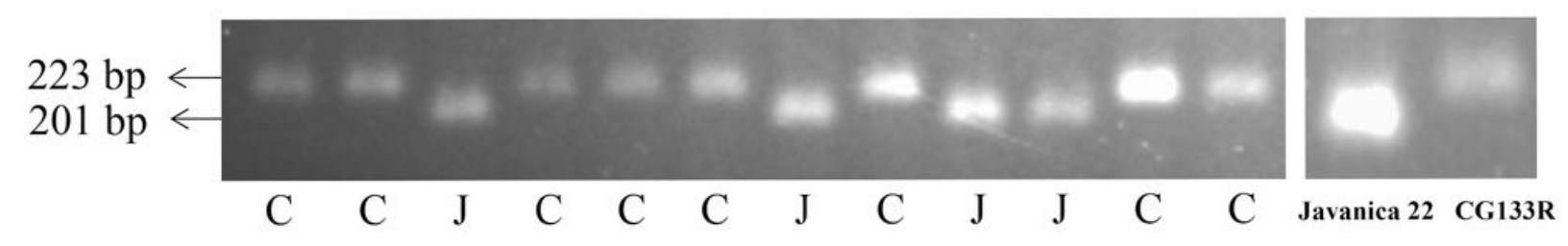

$S S I V-2 \mathrm{M} 2$

\section{Figure 1}

Polymorphism gene loci of starch synthesis-related genes between two parents and partial tested materials. $\mathrm{J}$ and I indicate genotype of Janavica 22, and C and II indicate genotype of CG133R. 


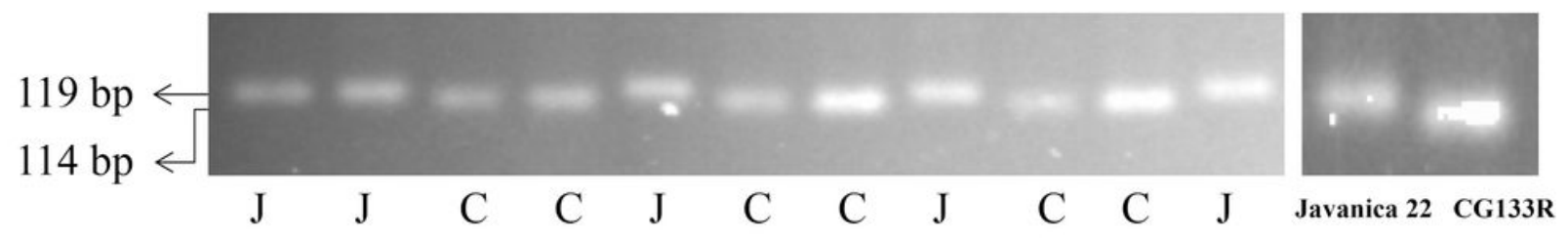

AGPlar M1

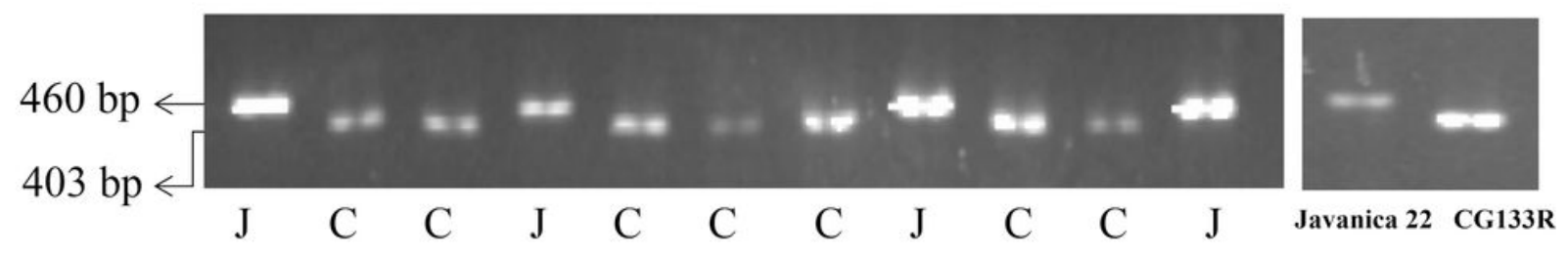

PCR-AccI
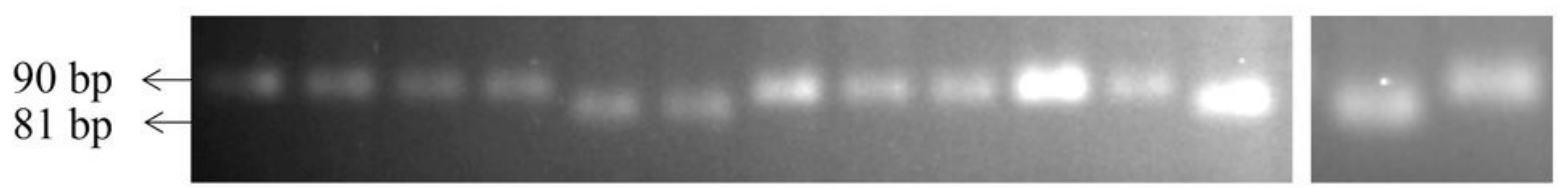

SSII-3 M1

$\begin{array}{lllllllllllllll}\text { II } & \text { II } & \text { II } & \text { II } & \text { I } & \text { I } & \text { II } & \text { II } & \text { II } & \text { II } & \text { II } & \text { I } & \text { Javanica } 22 & \text { CG133R }\end{array}$

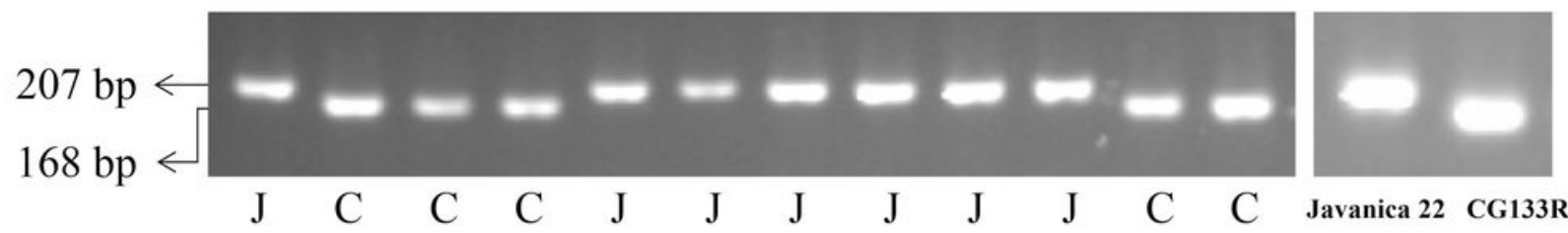

AGPsma 1

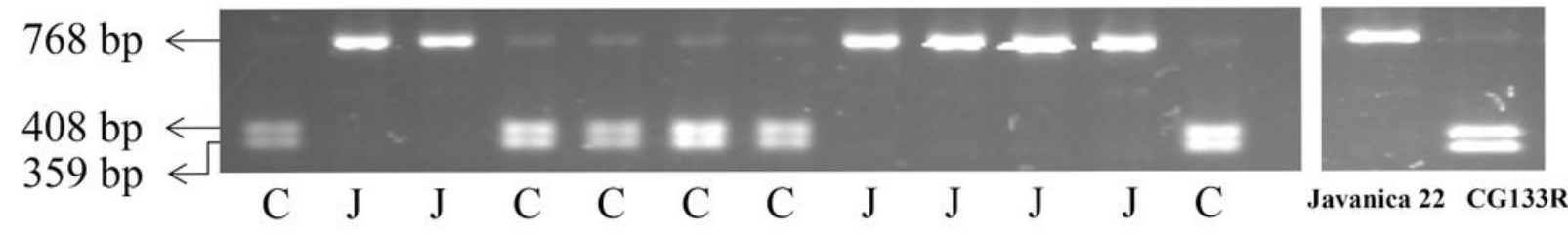

SSIII-1 t1

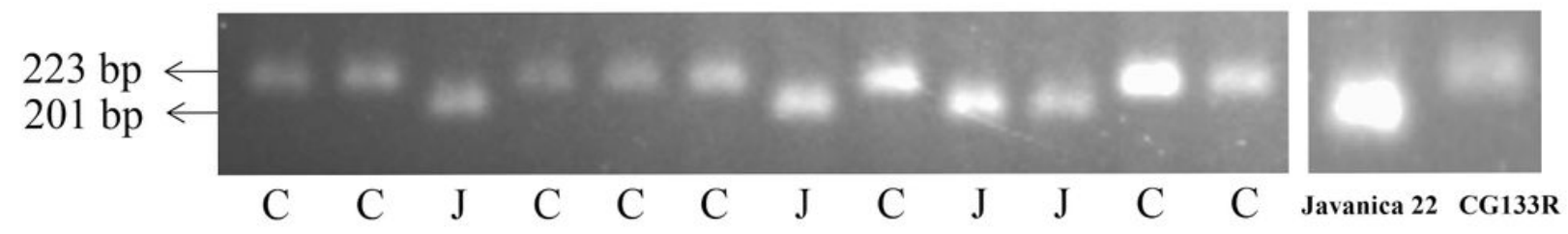

$S S I V-2 \mathrm{M} 2$

\section{Figure 1}

Polymorphism gene loci of starch synthesis-related genes between two parents and partial tested materials. J and I indicate genotype of Janavica 22, and C and II indicate genotype of CG133R. 

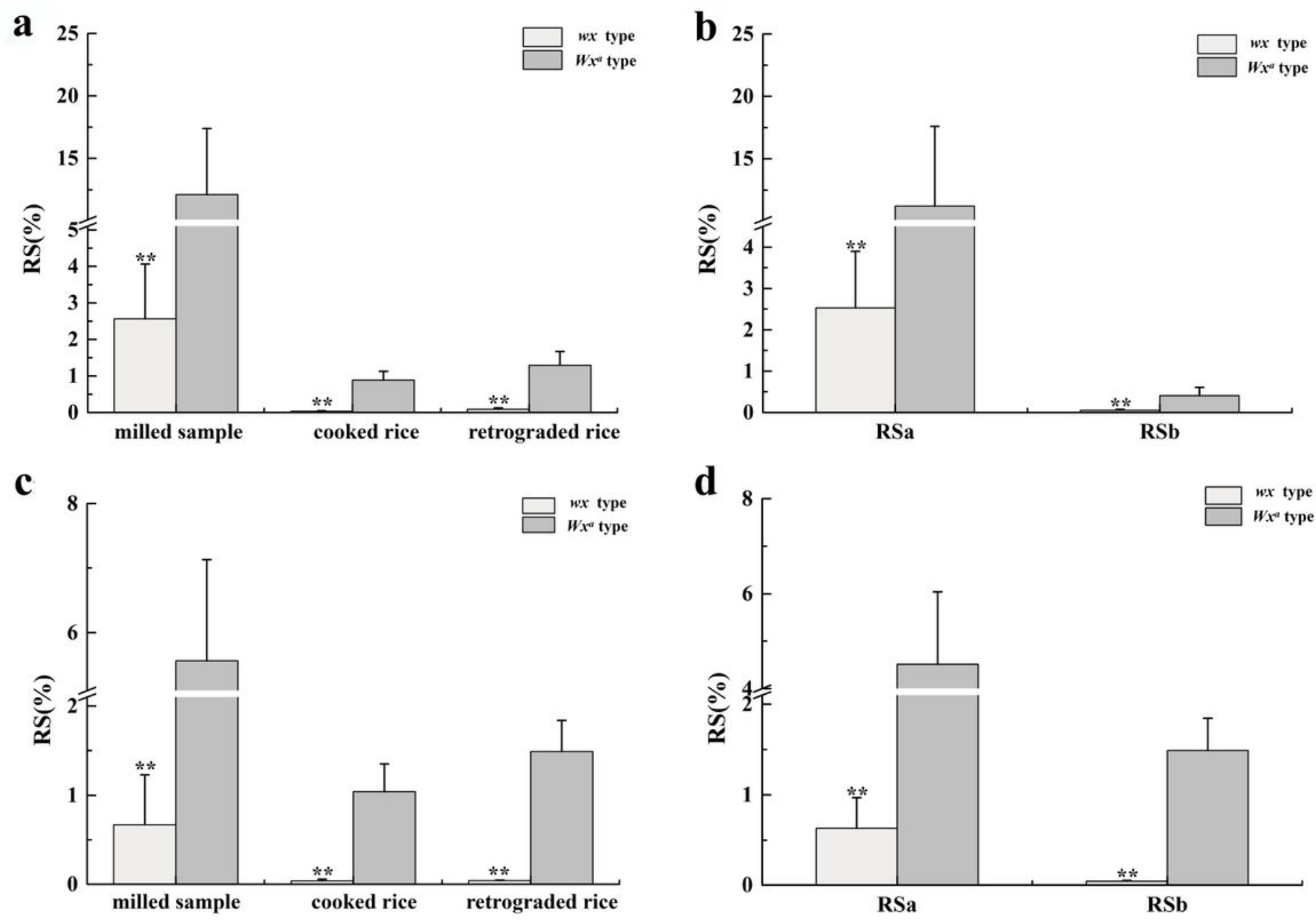

\section{Figure 2}

Comparison of RS among different Wx genotypes in RIL. wx type, Wxa type indicate homozygote for Janavica 22 and CG133R in Wx allele, respectively; RSa= RSm-RSc; RSb= RSr-RSc. a RS content in raw milled sample, cooked rice and retrograded rice at different Wx alleles in the RIL; $b$ RSa and RSb content at different Wx alleles in the RIL; C RS content in raw milled sample, cooked rice and retrograded rice at different Wx alleles in the 16 lines; $\mathrm{d}$ RSa and RSb content at different Wx alleles in the 16 lines. Each column represents the mean \pm standard deviation, $* *$ indicates $P<0.01$. 

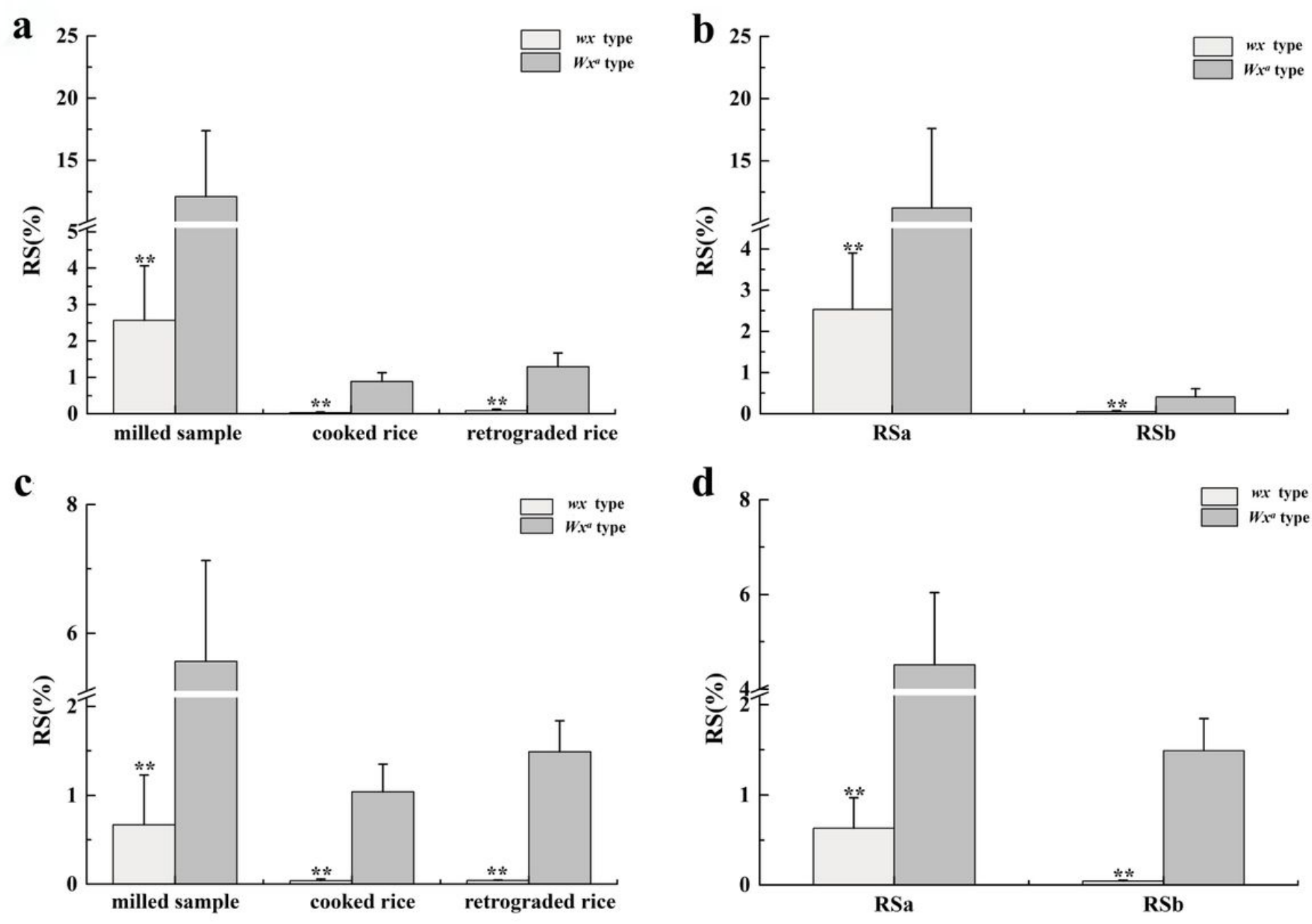

\section{Figure 2}

Comparison of RS among different Wx genotypes in RIL. wx type, Wxa type indicate homozygote for Janavica 22 and CG133R in Wx allele, respectively; RSa= RSm-RSc; RSb= RSr-RSc. a RS content in raw milled sample, cooked rice and retrograded rice at different Wx alleles in the RIL; $b$ RSa and RSb content at different Wx alleles in the RIL; C RS content in raw milled sample, cooked rice and retrograded rice at different Wx alleles in the 16 lines; $\mathrm{d}$ RSa and RSb content at different Wx alleles in the 16 lines. Each column represents the mean \pm standard deviation, $* *$ indicates $P<0.01$. 

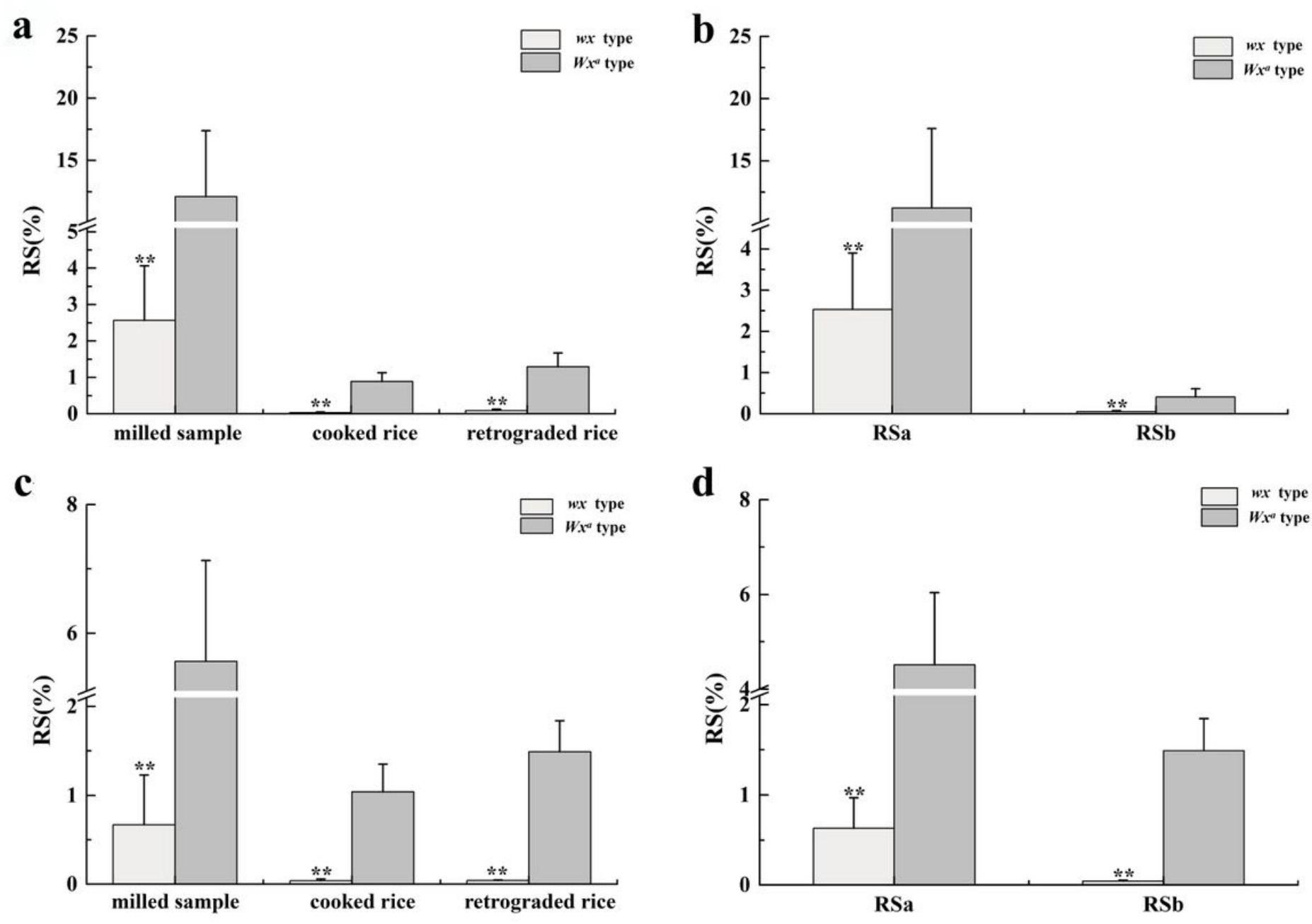

\section{Figure 2}

Comparison of RS among different Wx genotypes in RIL. wx type, Wxa type indicate homozygote for Janavica 22 and CG133R in Wx allele, respectively; RSa= RSm-RSc; RSb= RSr-RSc. a RS content in raw milled sample, cooked rice and retrograded rice at different Wx alleles in the RIL; $b$ RSa and RSb content at different Wx alleles in the RIL; C RS content in raw milled sample, cooked rice and retrograded rice at different Wx alleles in the 16 lines; $\mathrm{d}$ RSa and RSb content at different Wx alleles in the 16 lines. Each column represents the mean \pm standard deviation, $* *$ indicates $P<0.01$. 

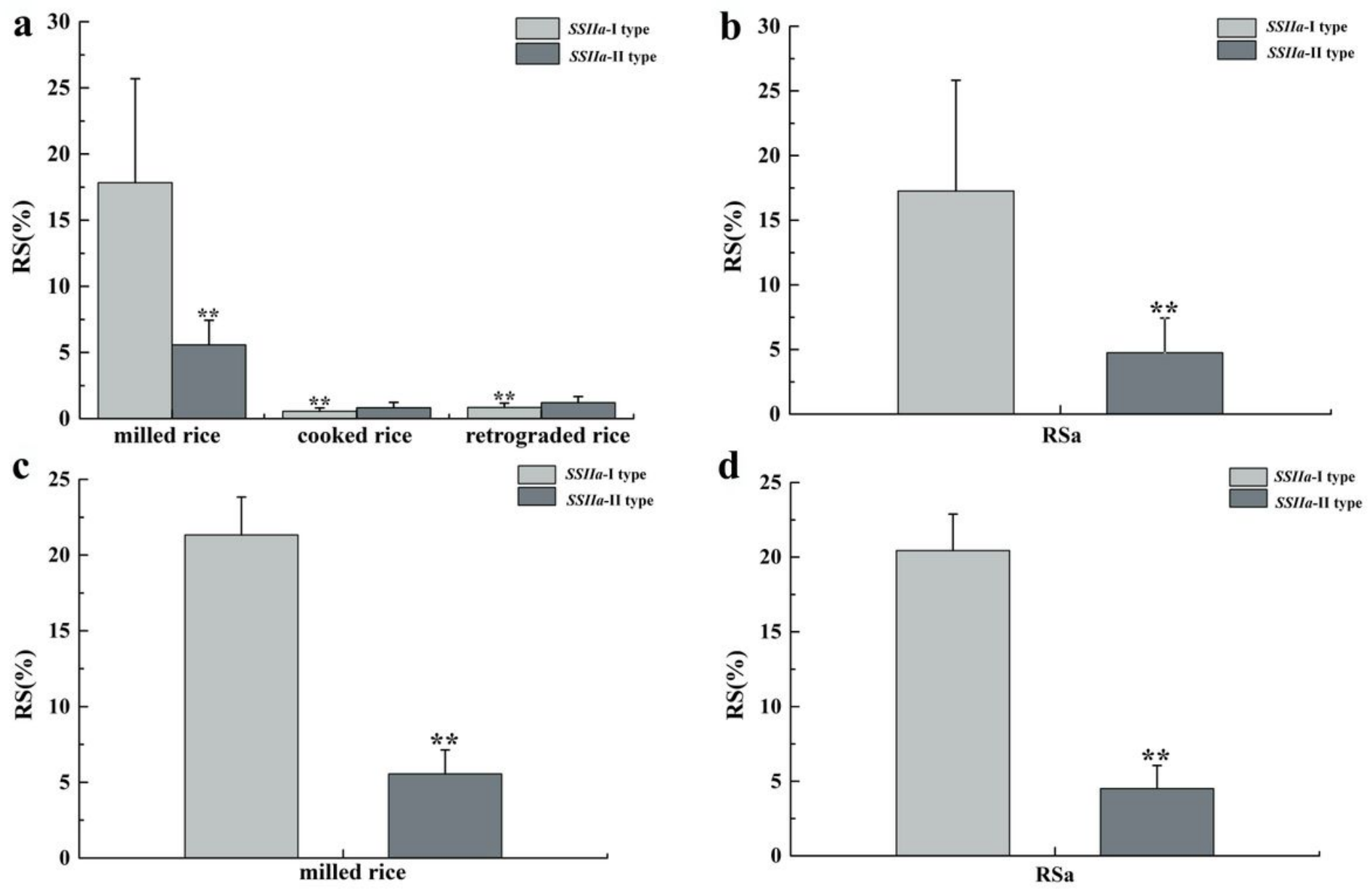

Figure 3

Comparison of RS among different SSIla genotypes in RIL. SSIla- $\bigotimes$, SSIla- $\bigotimes$ type indicate homozygote for Janavica 22 and CG133R in SSlla locus, respectively; RSa= RSm-RSc. a RS content in raw milled sample, cooked rice and retrograded rice at different SSIla alleles in the RIL; b RSa at different SSIla alleles in the RIL; c RS content in raw milled sample at different SSIla alleles in the 18 lines; $d$ RSa at different SSIla alleles in the 18 lines. Each column represents the mean \pm standard deviation, $* *$ indicates $P<0.01$. 

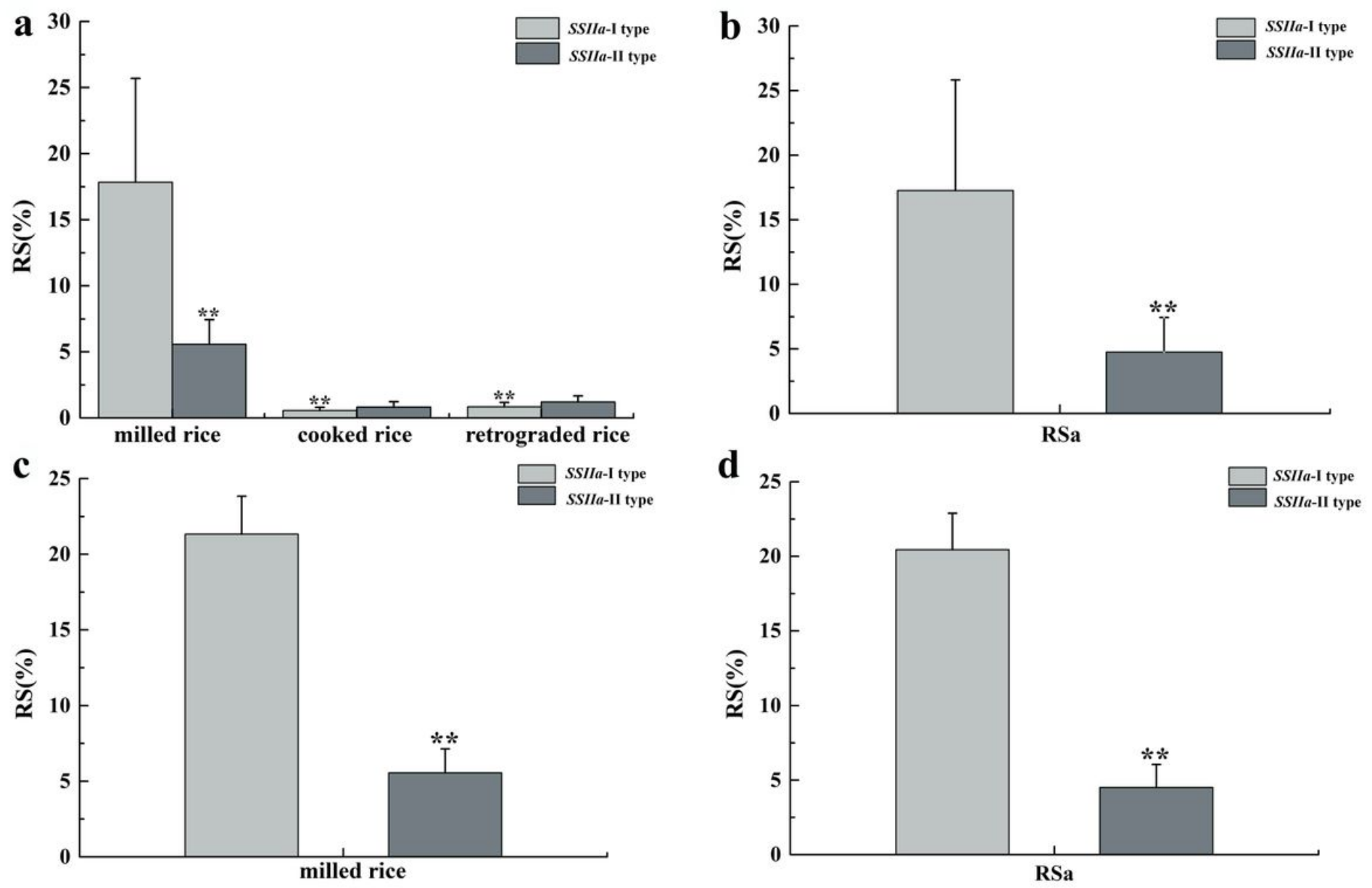

Figure 3

Comparison of RS among different SSIla genotypes in RIL. SSIla- $\bigotimes$, SSIla- $\bigotimes$ type indicate homozygote for Janavica 22 and CG133R in SSlla locus, respectively; RSa= RSm-RSc. a RS content in raw milled sample, cooked rice and retrograded rice at different SSIla alleles in the RIL; b RSa at different SSIla alleles in the RIL; c RS content in raw milled sample at different SSIla alleles in the 18 lines; $d$ RSa at different SSIla alleles in the 18 lines. Each column represents the mean \pm standard deviation, $* *$ indicates $P<0.01$. 

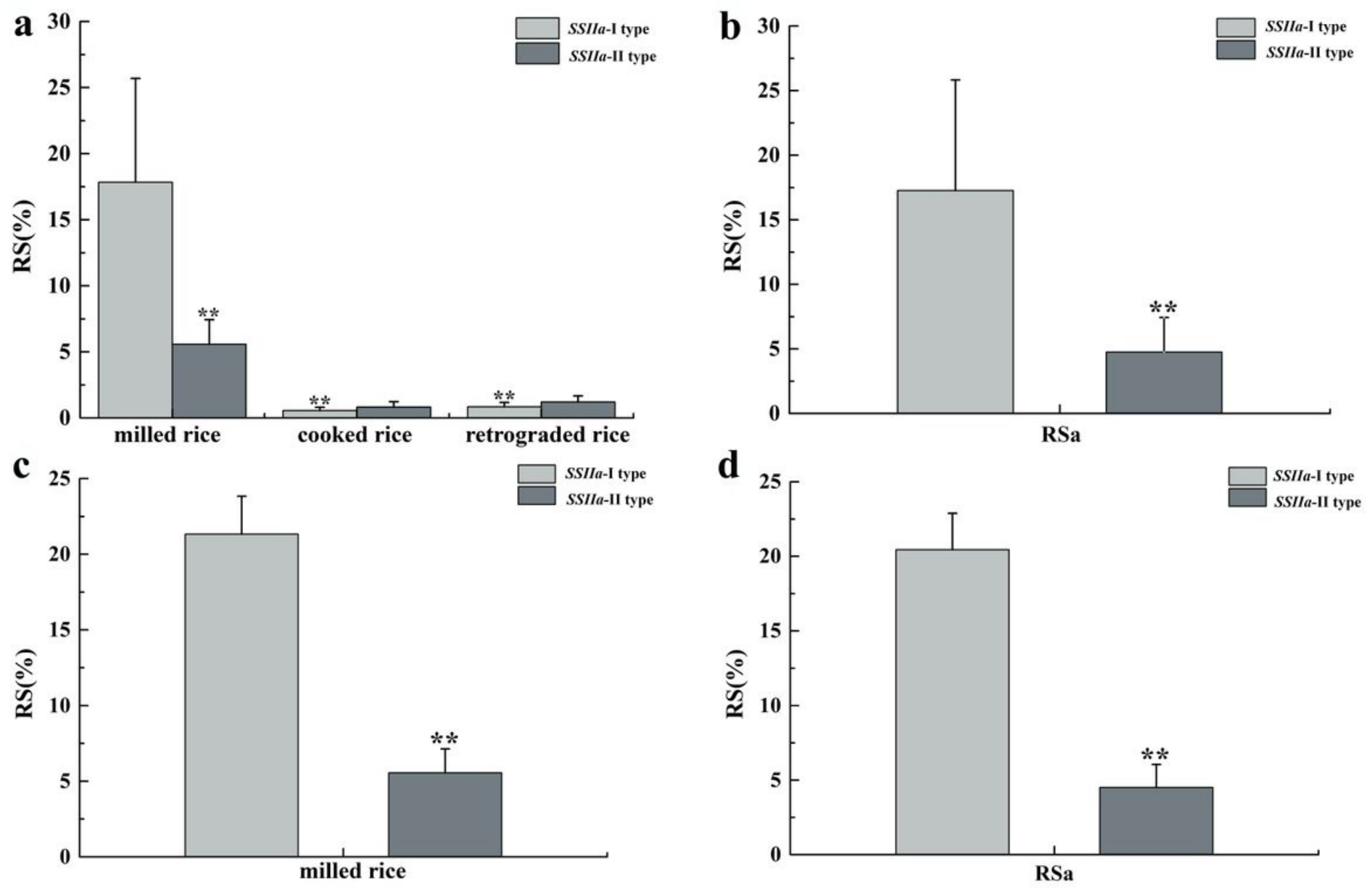

Figure 3

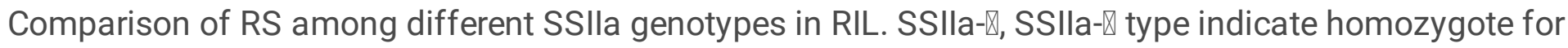
Janavica 22 and CG133R in SSlla locus, respectively; RSa= RSm-RSc. a RS content in raw milled sample, cooked rice and retrograded rice at different SSIla alleles in the RIL; b RSa at different SSIla alleles in the RIL; c RS content in raw milled sample at different SSIla alleles in the 18 lines; $d$ RSa at different SSIla alleles in the 18 lines. Each column represents the mean \pm standard deviation, $*$ indicates $\mathrm{P}<0.01$. 

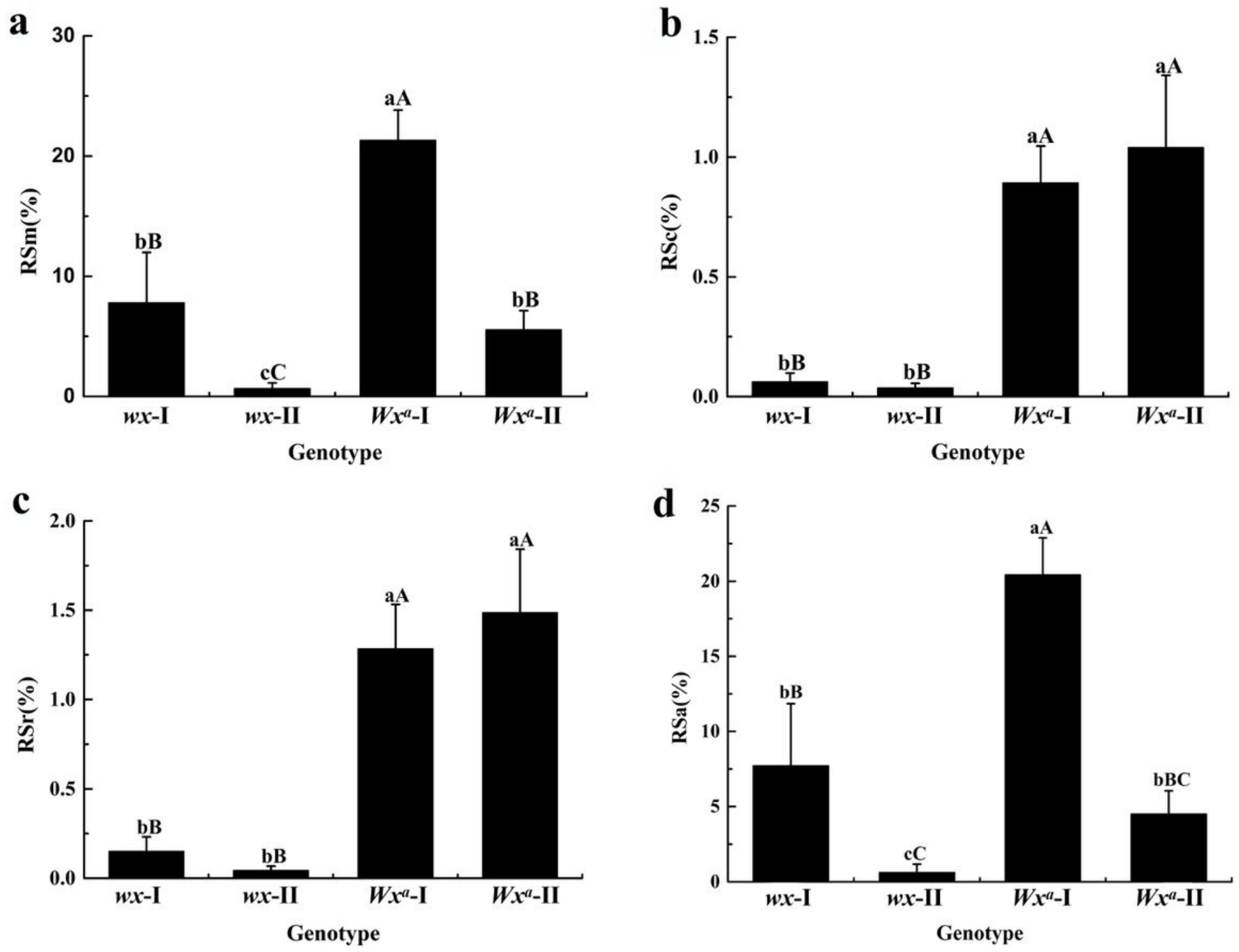

Figure 4

Comparison of RS for different combinations of Wx $\times$ SSIla. wx , Wxa type indicate homozygote for Janavica 22 and CG133R in Wx allele, respectively; I, II Type indicate homozygote for Janavica 22 and CG133R in SSIla locus, respectively; RSm, resistant starch content in raw milled sample; RSc, resistant starch content in cooked rice; RSr, resistant starch content in retrograded rice; $\mathrm{RSa}=\mathrm{RSm}-\mathrm{RSc}$. a RS content in raw milled sample at different combinations of Wx $\times$ SSIla; $b$ RS content in cooked rice at different combinations of Wx $\times$ SSIla. $c$ RS content in retrogaded rice at different combinations of $W x \times$ SSIla. $d$ RSa at different combinations of Wx $\times$ SSIla. Lowercase and capital letters above column denote significant at 0.05 and 0.01 levels, respectively. 

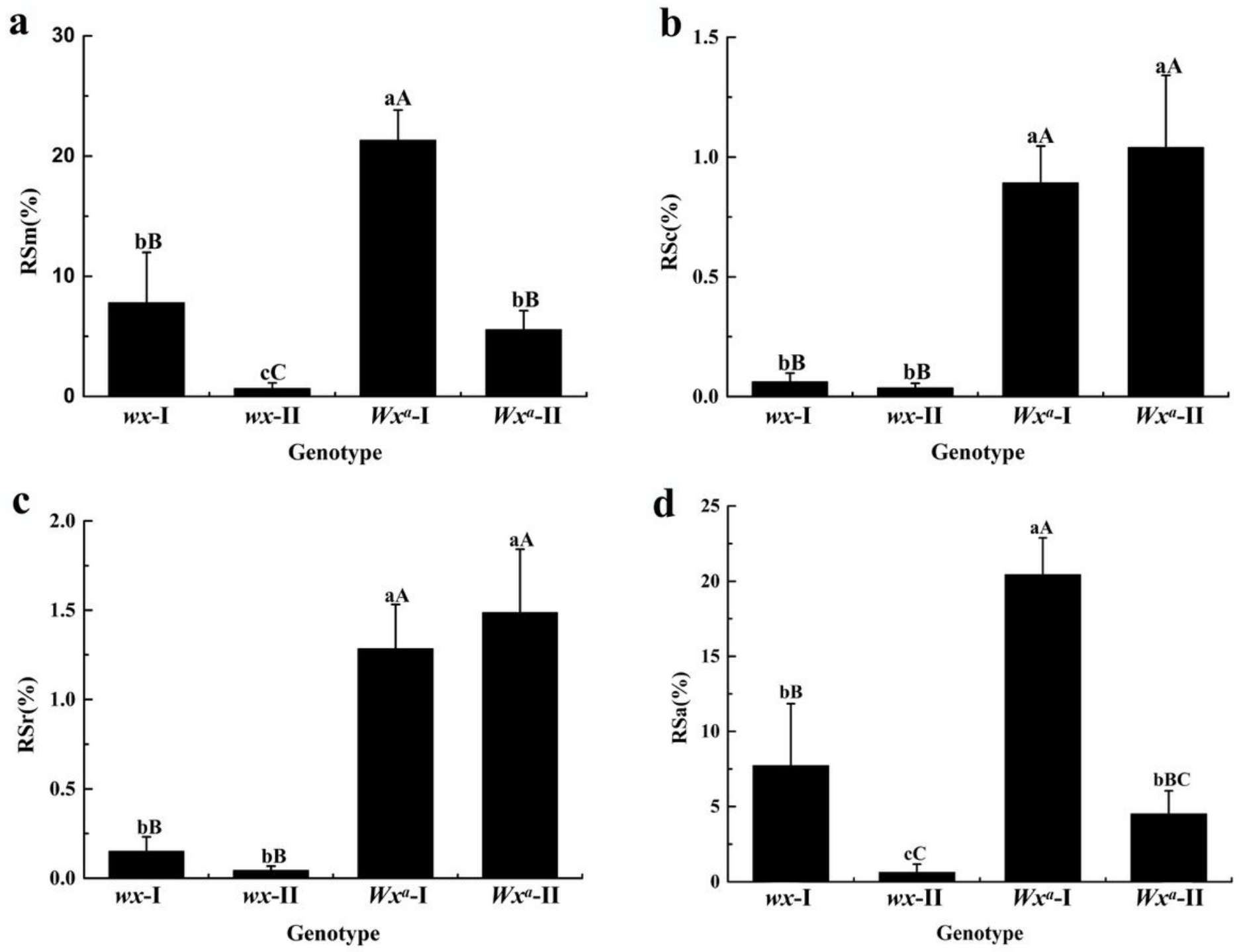

Figure 4

Comparison of RS for different combinations of Wx $\times$ SSIla. wx , Wxa type indicate homozygote for Janavica 22 and CG133R in Wx allele, respectively; I, II Type indicate homozygote for Janavica 22 and CG133R in SSIla locus, respectively; RSm, resistant starch content in raw milled sample; RSc, resistant starch content in cooked rice; RSr, resistant starch content in retrograded rice; $\mathrm{RSa}=\mathrm{RSm}-\mathrm{RSc}$. a RS content in raw milled sample at different combinations of Wx $\times$ SSIla; $b$ RS content in cooked rice at different combinations of Wx $\times$ SSIla. $c$ RS content in retrogaded rice at different combinations of $W x \times$ SSIla. $d$ RSa at different combinations of Wx $\times$ SSIla. Lowercase and capital letters above column denote significant at 0.05 and 0.01 levels, respectively. 

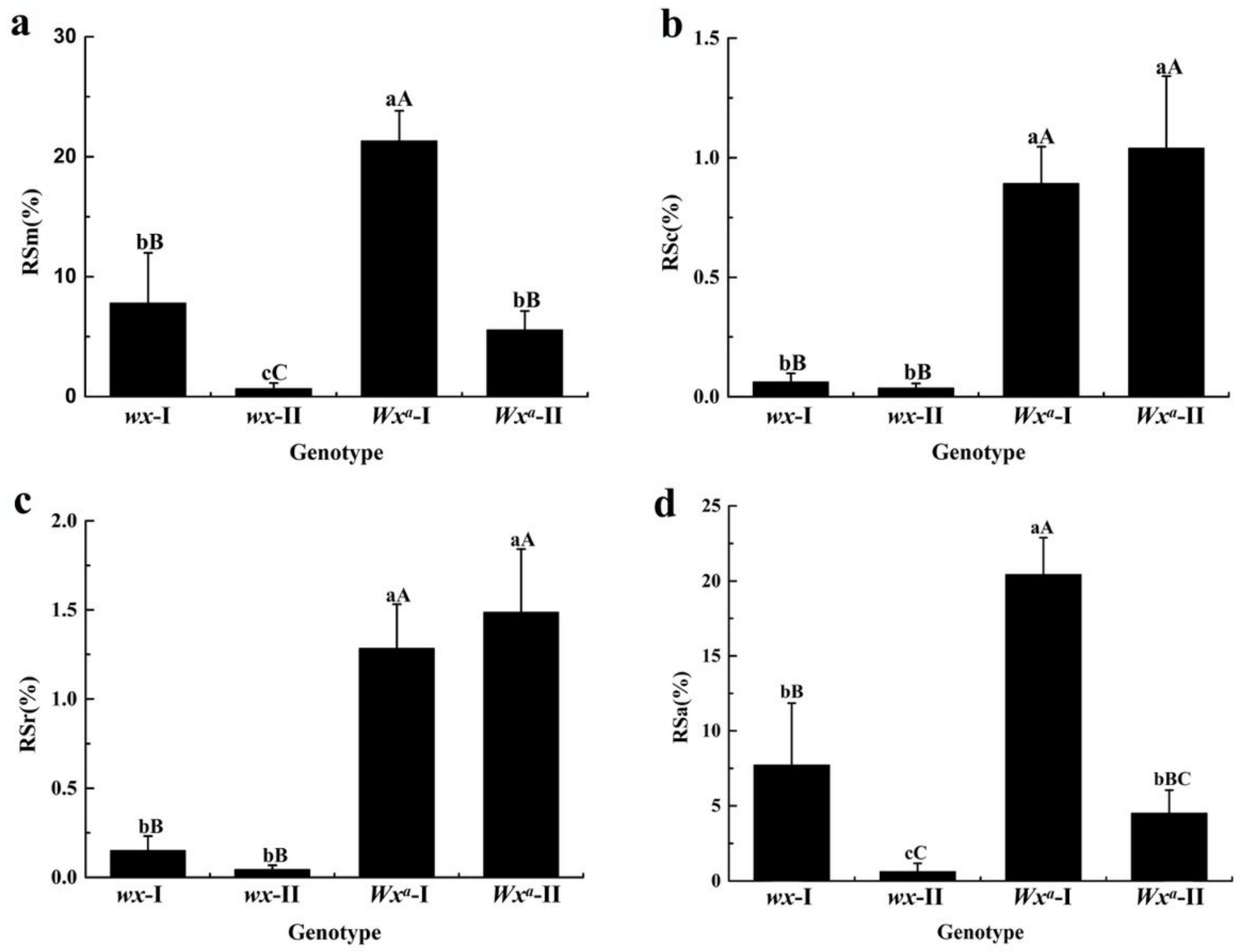

Figure 4

Comparison of RS for different combinations of Wx $\times$ SSIla. wx , Wxa type indicate homozygote for Janavica 22 and CG133R in Wx allele, respectively; I, II Type indicate homozygote for Janavica 22 and CG133R in SSIla locus, respectively; RSm, resistant starch content in raw milled sample; RSc, resistant starch content in cooked rice; RSr, resistant starch content in retrograded rice; $\mathrm{RSa}=\mathrm{RSm}-\mathrm{RSc}$. a RS content in raw milled sample at different combinations of Wx $\times$ SSIla; $b$ RS content in cooked rice at different combinations of Wx $\times$ SSIla. $c$ RS content in retrogaded rice at different combinations of $W x \times$ SSIla. $d$ RSa at different combinations of Wx $\times$ SSIla. Lowercase and capital letters above column denote significant at 0.05 and 0.01 levels, respectively. 


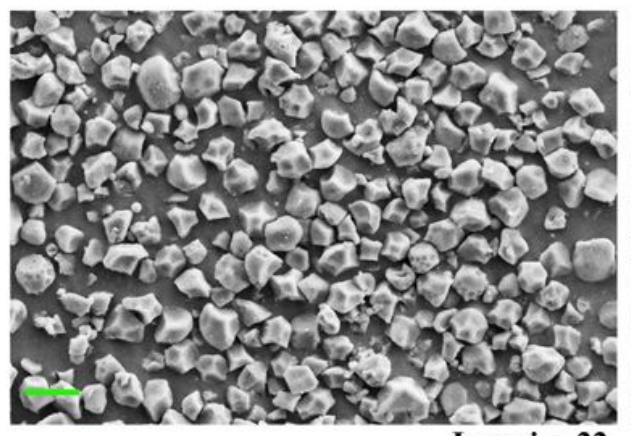
Javanica 22

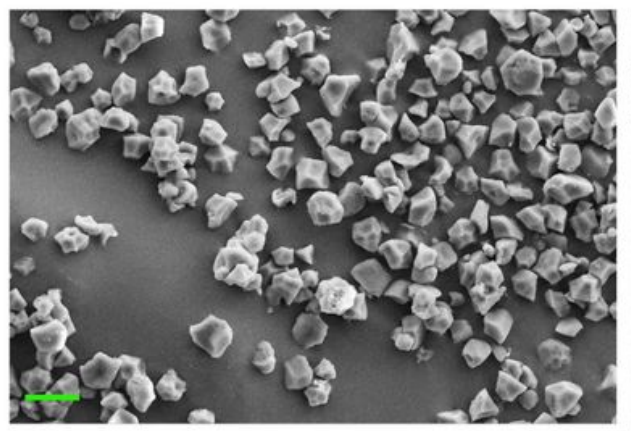

Bar $=10 \mu \mathrm{m}$

CG133R

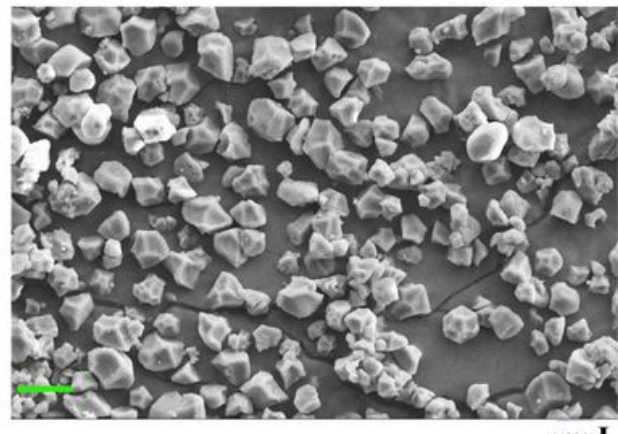
$w x-\mathrm{I}$

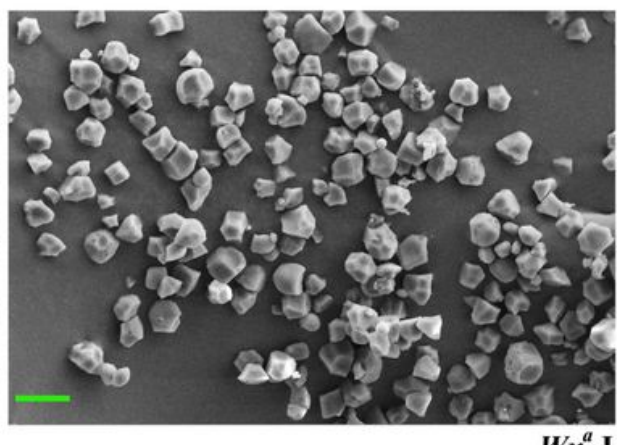

$W x^{a}-\mathbf{I}$

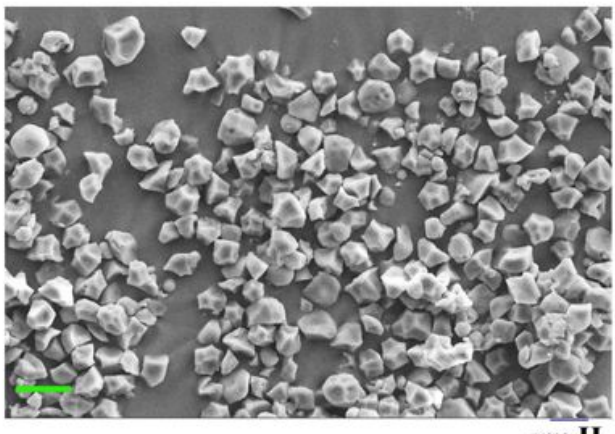

$w x-$ II

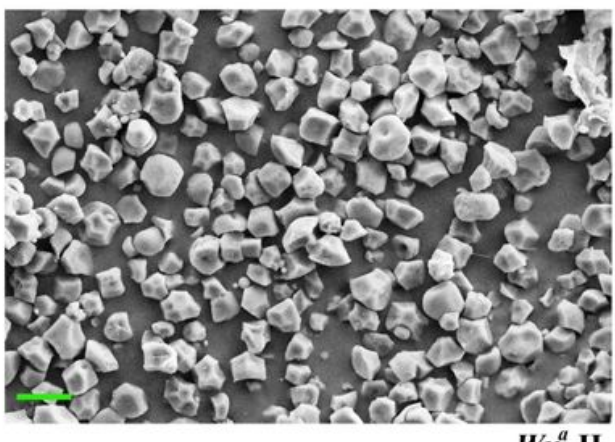

\section{Figure 5}

Scanning electron micrographs of starch granules from two parents and different Wx and SSIla combinations.

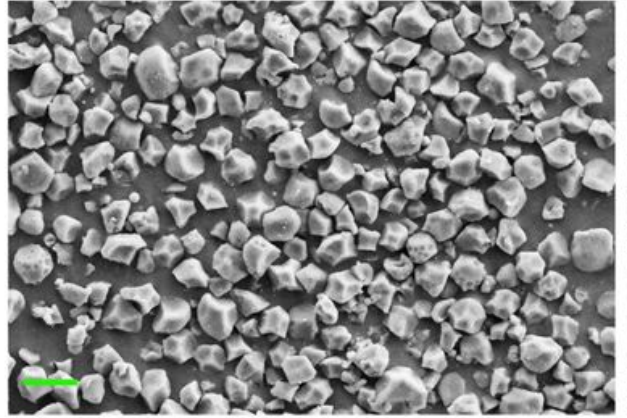

Javanica 22

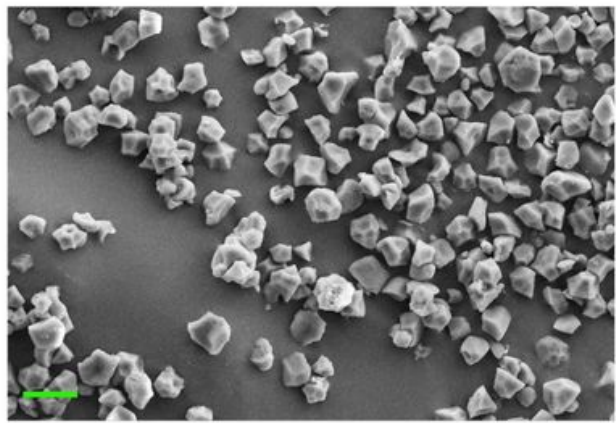

Bar $=10 \mu \mathrm{m}$

CG133R

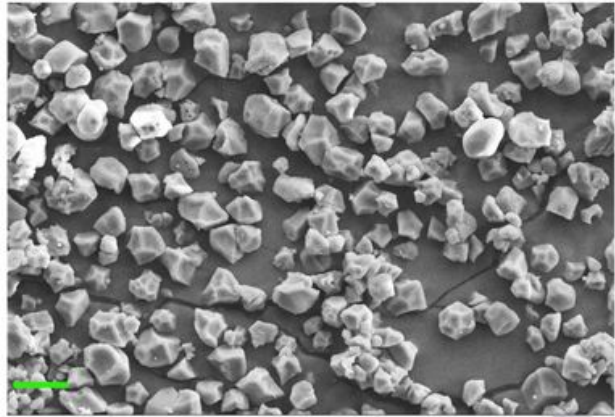

$w x-I$

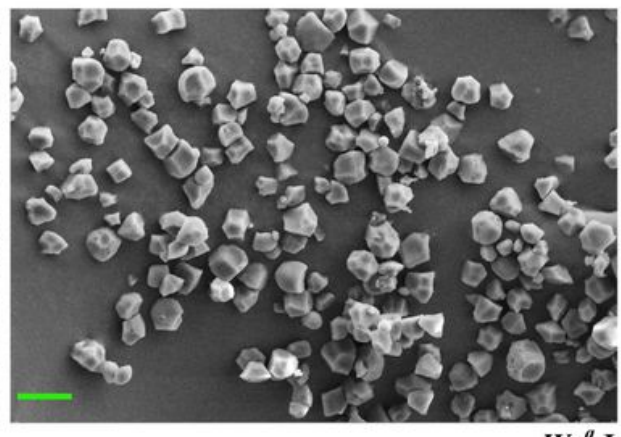

$W x^{a}-\mathrm{I}$

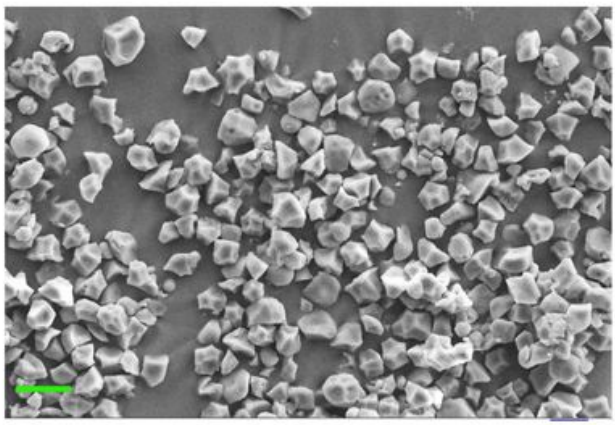

$w x-I I$

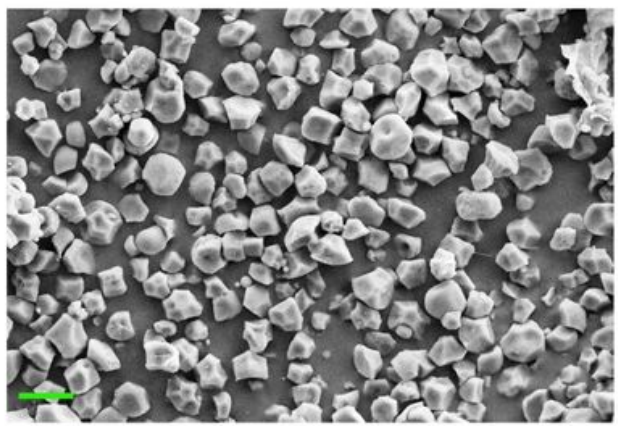

$W x^{a}-$ II

Figure 5 
Scanning electron micrographs of starch granules from two parents and different Wx and SSIla combinations.
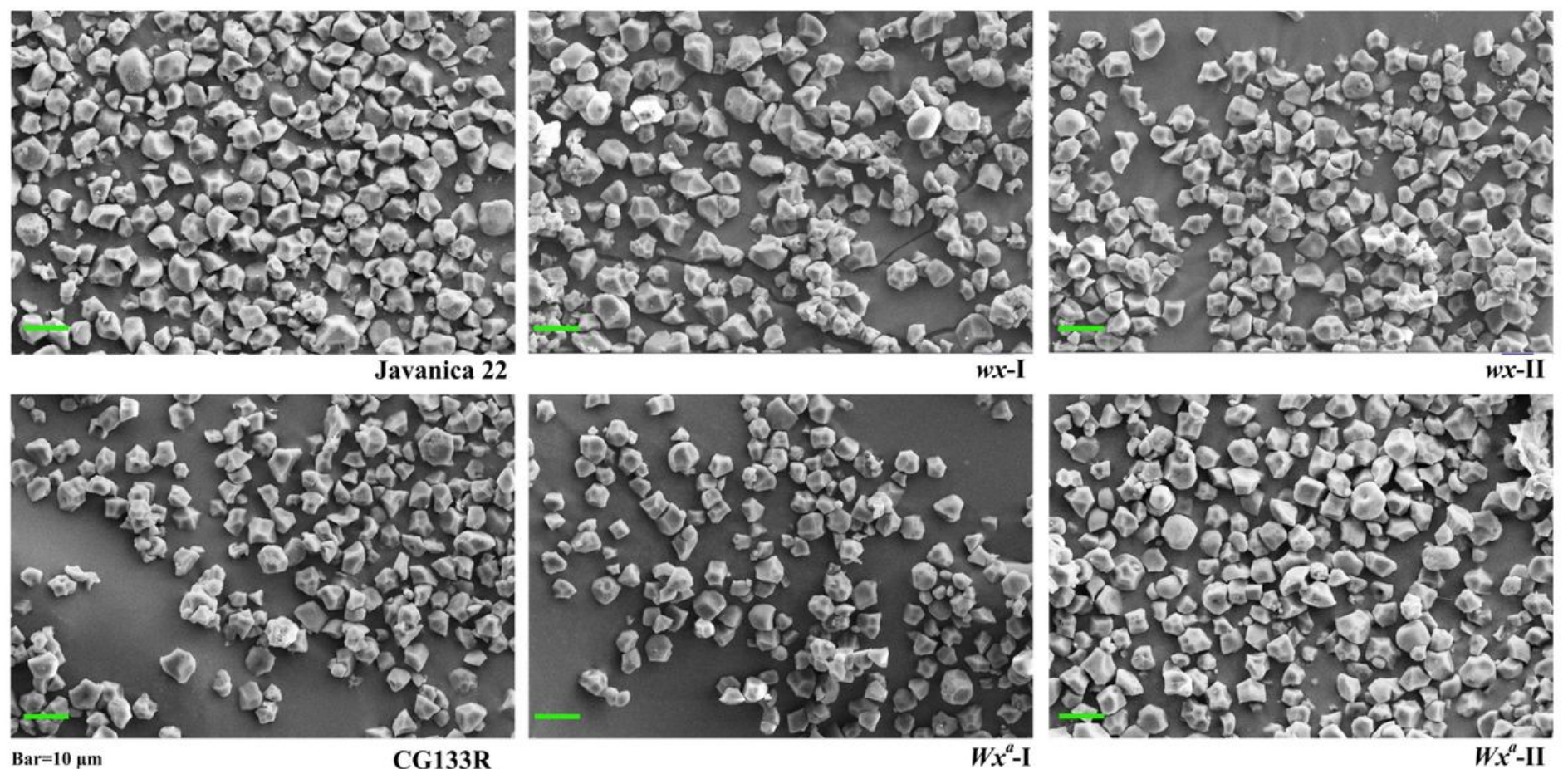

\section{Figure 5}

Scanning electron micrographs of starch granules from two parents and different Wx and SSIla combinations. 


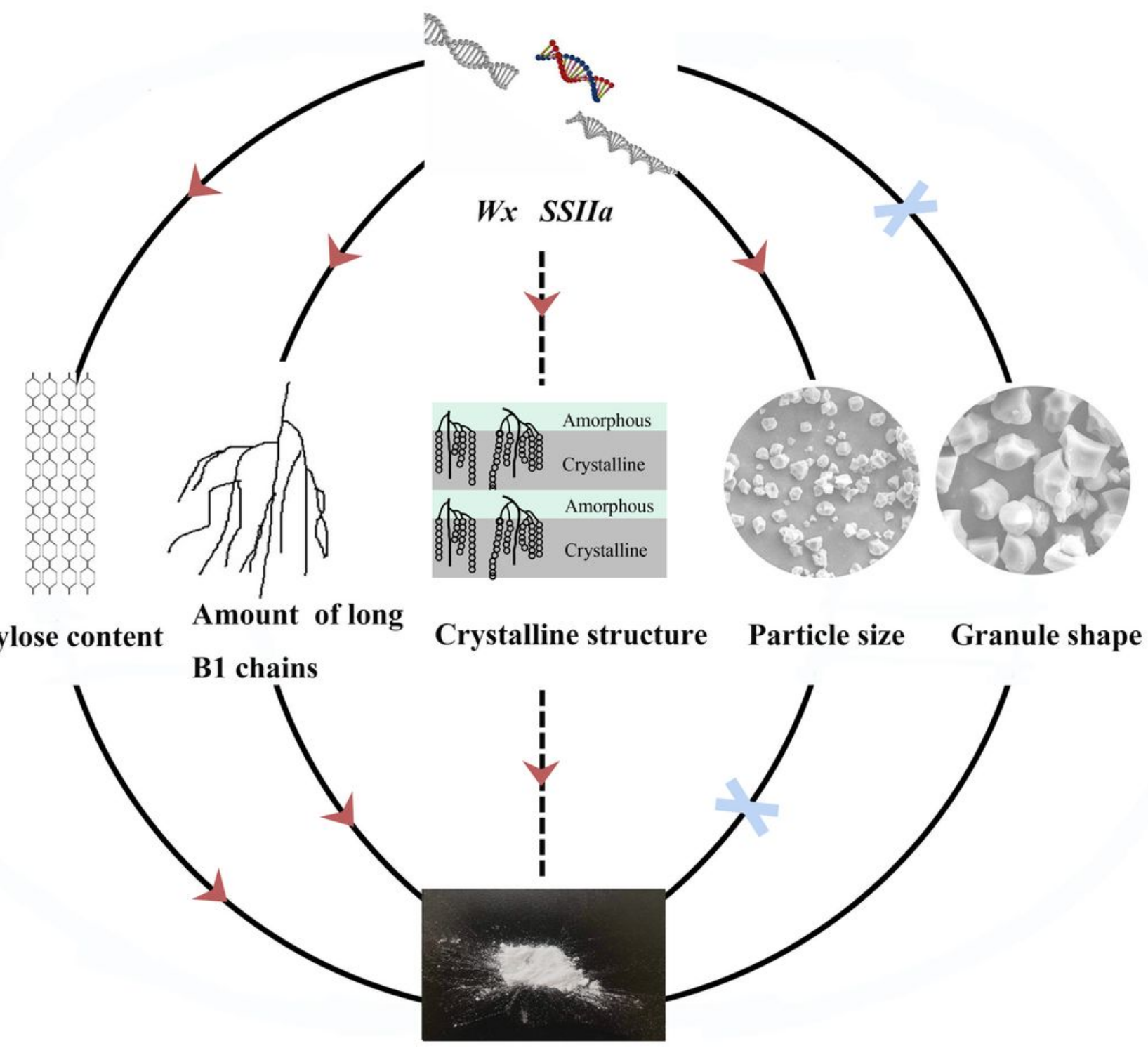

RSm RSc RSr RS2

Figure 6

The schematic diagram of Wx and SSlla regulating RS content in rice. We found that Wx affects RS in different processing rice through controlling amylose content, and SSIla affects RS content in raw starch and RS2 by regulating the amount of intermediate-size chains of amylopectin. The combine effects of Wx and SSIla can affect the starch particle size distribution, but not the granule shape. Unfortunately, there is no correlation between RS content and starch granule diameter. Thus, it imply that Wx and SSIla alleles may manipulate the RS content by controlling the crystalline structure of starch granules, which needs further verification. The solid line indicates the pathways of Wx and SSlla regulating RS content in rice; the dotted line represents a hypothetical path, which needs further confirmation. RSm, resistant starch content in raw milled sample; RSc, resistant starch content in cooked rice; RSr, resistant starch content in retrogradation. 


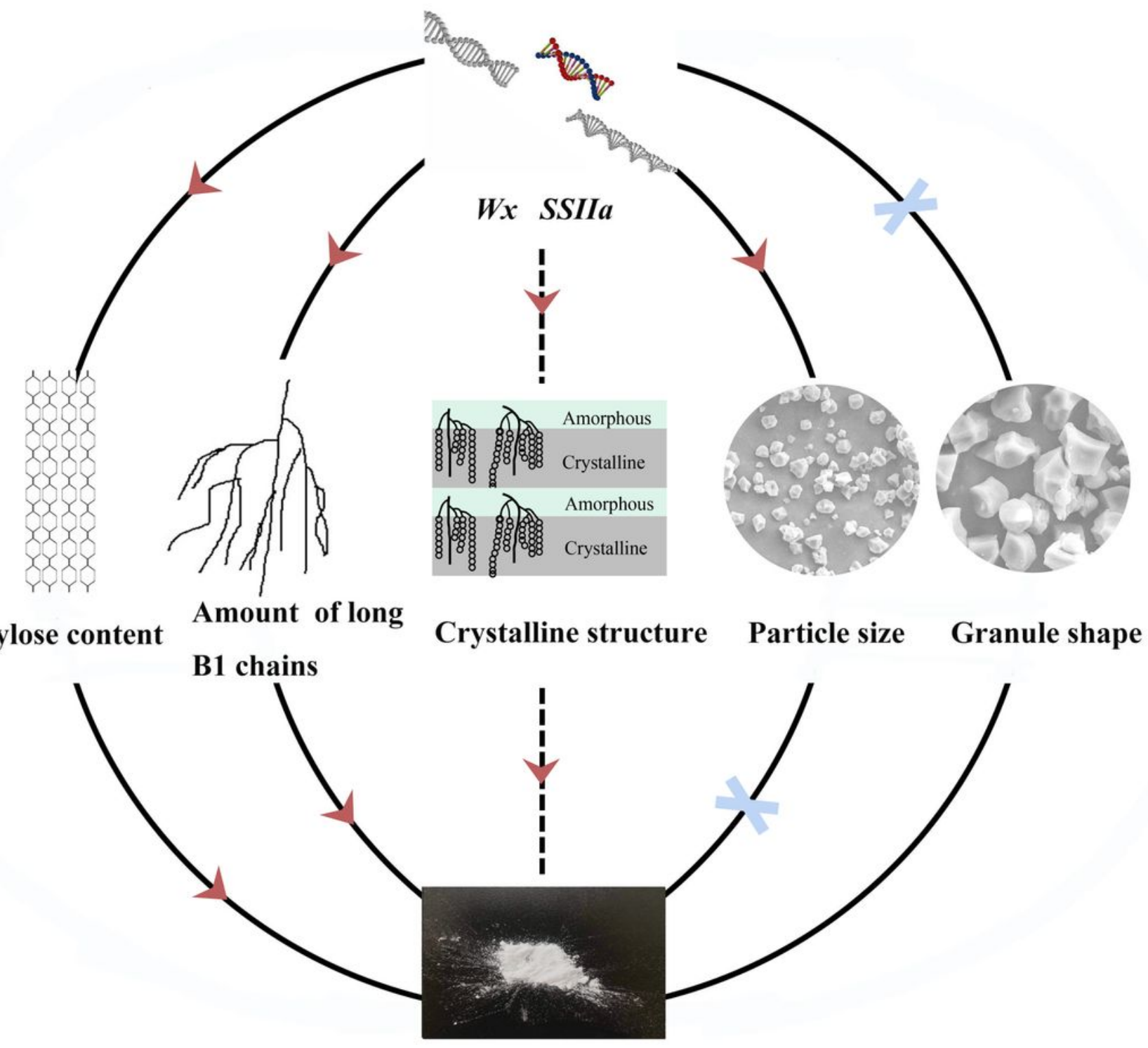

RSm RSc RSr RS2

Figure 6

The schematic diagram of Wx and SSlla regulating RS content in rice. We found that Wx affects RS in different processing rice through controlling amylose content, and SSIla affects RS content in raw starch and RS2 by regulating the amount of intermediate-size chains of amylopectin. The combine effects of Wx and SSIla can affect the starch particle size distribution, but not the granule shape. Unfortunately, there is no correlation between RS content and starch granule diameter. Thus, it imply that Wx and SSIla alleles may manipulate the RS content by controlling the crystalline structure of starch granules, which needs further verification. The solid line indicates the pathways of Wx and SSlla regulating RS content in rice; the dotted line represents a hypothetical path, which needs further confirmation. RSm, resistant starch content in raw milled sample; RSc, resistant starch content in cooked rice; RSr, resistant starch content in retrogradation. 


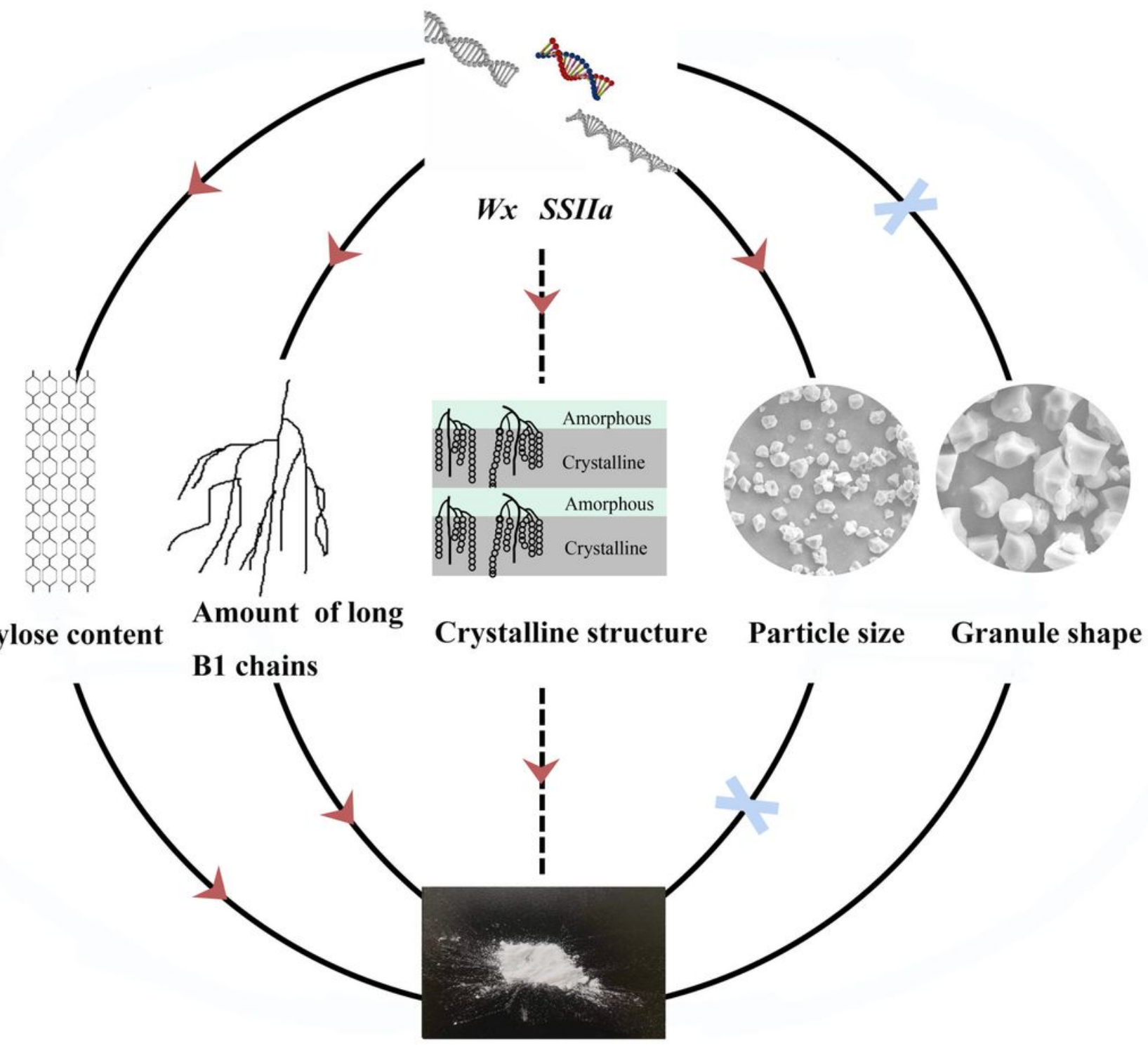

RSm RSc RSr RS2

Figure 6

The schematic diagram of Wx and SSlla regulating RS content in rice. We found that Wx affects RS in different processing rice through controlling amylose content, and SSIla affects RS content in raw starch and RS2 by regulating the amount of intermediate-size chains of amylopectin. The combine effects of Wx and SSIla can affect the starch particle size distribution, but not the granule shape. Unfortunately, there is no correlation between RS content and starch granule diameter. Thus, it imply that Wx and SSIla alleles may manipulate the RS content by controlling the crystalline structure of starch granules, which needs further verification. The solid line indicates the pathways of Wx and SSlla regulating RS content in rice; the dotted line represents a hypothetical path, which needs further confirmation. RSm, resistant starch content in raw milled sample; RSc, resistant starch content in cooked rice; RSr, resistant starch content in retrogradation. 


\section{Supplementary Files}

This is a list of supplementary files associated with this preprint. Click to download.

- Additionalfile1.doc

- Additionalfile1.doc

- Additionalfile1.doc

- Additionalfile2.doc

- Additionalfile2.doc

- Additionalfile2.doc 